\title{
SIMPLE LENGTH RIGIDITY FOR KLEINIAN SURFACE GROUPS AND APPLICATIONS
}

\author{
MARTIN BRIDGEMAN AND RICHARD D. CANARY
}

\begin{abstract}
We prove that a Kleinian surface groups is determined, up to conjugacy in the isometry group of $\mathbb{H}^{3}$, by its simple marked length spectrum. As a first application, we show that a discrete faithful representation of the fundamental group of a compact, acylindrical, hyperblizable 3 -manifold $M$ is similarly determined by the translation lengths of images of elements of $\pi_{1}(M)$ represented by simple curves on the boundary of $M$. As a second application, we show the group of diffeomorphisms of quasifuchsian space which preserve the renormalized intersection number is generated by the (extended) mapping class group and complex conjugation.
\end{abstract}

\section{INTRODUCTION}

We show that if $\rho_{1}$ and $\rho_{2}$ are two discrete, faithful representations of a surface group $\pi_{1}(S)$ into $\operatorname{PSL}(2, \mathbb{C})$ with the same simple marked length spectrum, then $\rho_{1}$ is either conjugate to $\rho_{2}$ or its complex conjugate. (Two such representations have the same simple marked length spectrum if whenever $\alpha \in \pi_{1}(S)$ is represented by a simple closed curve, then the images of $\alpha$ have the same translation length. The complex conjugate of a representation is obtained by conjugating the representation by $z \rightarrow \bar{z}$.) Marché and Wolff [21, Sec. 3] have exhibited non-elementary representations of a closed surface group of genus two into $\operatorname{PSL}(2, \mathbb{R})$ with the same simple marked length spectrum which do not have the same marked length spectrum, so the corresponding statement does not hold for non-elementary representations.

We give two applications of our main result. First, if $M$ is a compact, acylindrical, hyperbolizable 3-manifold, we show that if $\rho_{1}$ and $\rho_{2}$ are discrete faithful representations of $\pi_{1}(M)$ into $\operatorname{PSL}(2, \mathbb{C})$ such that translation lengths of the images of elements of $\pi_{1}(M)$ corresponding to simple curves in the boundary of $M$ agree, then $\rho_{1}$ is either conjugate to $\rho_{2}$ or its complex conjugate. For our second application we consider the renormalized intersection number, first defined by Burger [9] and further studied by Bridgeman-Taylor [7]. Bridgeman [5] (see also [6]) showed that the Hessian of the renormalized intersection number gives rise to a path metric on quasifuchsian space $Q F(S)$. We show that the group of diffeomorphisms of $Q F(S)$ which preserve the renormalized intersection number is

Date: September 8, 2015.

Bridgeman was partially suppported by grant DMS-1500545 and Canary was partially supported by grant DMS-1306992, from the National Science Foundation. The authors also acknowledge support from U.S. National Science Foundation grants DMS 1107452, 1107263, 1107367 "RNMS: GEometric structures And Representation varieties" (the GEAR Network). 
generated by the (extended) mapping class group and the involution of $Q F(S)$ determined by complex conjugation.

1.1. Simple length rigidity for quasifuchsian surface groups. A Kleinian surface group is a discrete, faithful representation $\rho: \pi_{1}(S) \rightarrow \operatorname{PSL}(2, \mathbb{C})$ where $S$ is a closed, connected, orientable surface of genus at least two. If $\alpha \in \pi_{1}(S)$, then let $\ell_{\rho}(\alpha)$ denote the translation of length of $\rho(\alpha)$, or equivalently the length of the closed geodesic in the homotopy class of $\alpha$ in the quotient hyperbolic 3-manifold $\mathbb{H}^{3} / \rho\left(\pi_{1}(S)\right)$. We say that two Kleinian surface groups $\rho_{1}: \pi_{1}(S) \rightarrow \operatorname{PSL}(2, \mathbb{C})$ and $\rho_{2}: \pi_{1}(S) \rightarrow \operatorname{PSL}(2, \mathbb{C})$ have the same marked length spectrum if $\ell_{\rho_{1}}(\alpha)=\ell_{\rho_{2}}(\alpha)$ for all $\alpha \in \pi_{1}(S)$. Similarly, we say that $\rho_{1}$ and $\rho_{2}$ have the same simple marked length spectrum if $\ell_{\rho_{1}}(\alpha)=\ell_{\rho_{2}}(\alpha)$ whenever $\alpha$ has a representative on $S$ which is a simple closed curve. If $\rho: G \rightarrow \operatorname{PSL}(2, \mathbb{C})$ is a representation we define its complex conjugate $\bar{\rho}_{2}$ to be the representation obtained by conjugating by $z \rightarrow \bar{z}$.

Theorem 1.1. (Simple length rigidity for Kleinian surface groups) If $S$ is a closed, connected, orientable surface of genus at least two, and $\rho_{1}: \pi_{1}(S) \rightarrow \operatorname{PSL}(2, \mathbb{C})$ and $\rho_{2}: \pi_{1}(S) \rightarrow \operatorname{PSL}(2, \mathbb{C})$ are Kleinian surface groups with the same simple marked length spectrum, then $\rho_{1}$ is conjugate to either $\rho_{2}$ or $\bar{\rho}_{2}$.

Since the full isometry group of $\mathbb{H}^{3}$ may be identified with the group generated by $\operatorname{PSL}(2, \mathbb{C})$, regarded as the group of fractional linear transformations, and $z \rightarrow \bar{z}$, one may reformulate our main result as saying that two Kleinian surface groups with the same simple marked length spectrum are conjugate in the isometry group of $\mathbb{H}^{3}$.

As a consequence of our main theorem and basic algebraic results, we see that it suffices to consider finitely many simple curves.

Corollary 1.2. If $S$ is a closed, connected, orientable surface of genus at least two, then there exists finitely many simple curves $\left\{\alpha_{1}, \ldots, \alpha_{n}\right\}$ on $S$ such that if $\rho_{1}: \pi_{1}(S) \rightarrow \operatorname{PSL}(2, \mathbb{C})$ and $\rho_{2}: \pi_{1}(S) \rightarrow \operatorname{PSL}(2, \mathbb{C})$ are Kleinian surface groups and $\ell_{\rho_{1}}\left(\alpha_{j}\right)=\ell_{\rho_{2}}\left(\alpha_{j}\right)$ for all $j=1, \ldots, n$, then $\rho_{1}$ is conjugate to either $\rho_{2}$ or $\bar{\rho}_{2}$.

Historical remarks: It is a classical consequence of the Fenchel-Nielsen coordinates for Teichmüller space that there are finitely many simple curves on $S$ whose lengths determine a Fuchsian (i.e. discrete and faithful) representation of $\pi_{1}(S)$ into $\operatorname{PSL}(2, \mathbb{R})$ up to conjugacy in $\operatorname{PGL}(2, \mathbb{R})$, which we may identify with the isometry group of $\mathbb{H}^{2}$. However, Marché and Wolff $[21$, Sec. 3] showed that there exist non-Fuchsian representations of the fundamental group of a surface of genus two into $\operatorname{PSL}(2, \mathbb{R})$ with the same simple marked length spectrum which do not have the same marked length spectrum. The representations constructed by Marché and Wolff do not lift to $\operatorname{SL}(2, \mathbb{R})$, so do not lie in the same component of the representation variety as the discrete faithful representations.

Kourounitis [19] showed that there are finitely many simple curves on $S$ whose complex lengths (see section 2 for a discussion of complex length) determine a quasifuchsian surface group up to conjugacy in $\operatorname{PSL}(2, \mathbb{C})$. Culler and Shalen [13, Prop. 1.4.1] showed that there are finitely many curves whose traces determine a non-elementary representation 
into $\mathrm{SL}(2, \mathbb{C})$, up to conjugacy in $\mathrm{SL}(2, \mathbb{C})$, while Charles-Marché $[11$, Thm. 1.1] showed that one may choose the finite collection to consist of simple closed curves. $\operatorname{Kim}[17,18]$ showed that there are finitely many curves on $S$ whose lengths determine a non-elementary representation of $\pi_{1}(S)$ into $\operatorname{PSL}(2, \mathbb{C})$ up to conjugacy in the isometry group of $\mathbb{H}^{3}$. The example of Marché and Wolff [21] shows that the curves produced by Kim's techniques cannot all be simple.

1.2. Simple length rigidity for acylindrical hyperbolic 3-manifolds. A compact, orientable 3-manifold $M$ with non-empty boundary is said to be hyperbolizable if its interior admits a complete hyperbolic metric, which implies that there exists a discrete, faithful representation of $\pi_{1}(M)$ into $\operatorname{PSL}(2, \mathbb{C})$. A compact, hyperbolizable 3-manifold is said to be acylindrical if every $\pi_{1}$-injective proper map of an annulus into $M$ is properly homotopic into the boundary of $M$. (Recall that a map of a surface into a 3-manifold is said to be proper if it maps the boundary of the surface into the boundary of 3-manifold and that a proper homotopy is a homotopy through proper maps.)

In this setting, we use Theorem 1.1 show that a discrete, faithful representation of $\pi_{1}(M)$ into $\operatorname{PSL}(2, \mathbb{C})$ is determined, up to conjugacy in the isometry group of $\mathbb{H}^{3}$, by the translation lengths of images of simple curves in the boundary $\partial M$ of $M$.

Theorem 1.3. If $M$ is a compact, acylindrical, hyperbolizable 3-manifold, and $\rho_{1}: \pi_{1}(M) \rightarrow \operatorname{PSL}(2, \mathbb{C})$ and $\rho_{2}: \pi_{1}(M) \rightarrow \operatorname{PSL}(2, \mathbb{C})$ are two discrete faithful representations, such that $\ell_{\rho_{1}}(\alpha)=$ $\ell_{\rho_{2}}(\alpha)$ if $\alpha \in \pi_{1}(M)$ is represented by a simple closed curve on $\partial M$, then $\rho_{1}$ is conjugate to either $\rho_{2}$ or $\bar{\rho}_{2}$.

1.3. Isometries of the renormalized intersection number. Burger [9] introduced a renormalized intersection number between convex cocompact representations into rank one Lie groups. Bridgeman and Taylor [7] extensively studied this renormalized intersection number in the setting of quasifuchsian representation. We say that $\rho: \pi_{1}(S) \rightarrow \operatorname{PSL}(2, \mathbb{C})$ is quasifuchsian if it is topologically conjugate, in terms of its action on $\widehat{\mathbb{C}}$, to a Fuchsian representation into $\operatorname{PSL}(2, \mathbb{R})$. If $T>0$ we let

$$
R_{T}(\rho)=\left\{[\alpha] \in\left[\pi_{1}(S)\right] \mid \ell_{\rho}(\alpha) \leq T\right\}
$$

where $\left[\pi_{1}(S)\right]$ is the set of conjugacy classes in $\pi_{1}(S)$. We define the entropy

$$
h(\rho)=\lim \sup \frac{\log \left(\#\left(R_{T}(\rho)\right)\right)}{T}
$$

of a quasifuchsian representation $\rho$. Sullivan [32] showed that $h(\rho)$ is the Hausdorff dimension of the limit set of $\rho\left(\pi_{1}(S)\right)$.

Let $Q F(S)$ denote the space of $\operatorname{PSL}(2, \mathbb{C})$-conjugacy classes of quasifuchsian representations. Bers [1] showed that $Q F(S)$ is an analytic manifold which may be naturally identified with $\mathcal{T}(S) \times \mathcal{T}(S)$. If $\rho_{1}, \rho_{2} \in Q F(S)$, the renormalized intersection number of $\rho_{1}$ and $\rho_{2}$ is given by

$$
J\left(\rho_{1}, \rho_{2}\right)=\frac{h\left(\rho_{2}\right)}{h\left(\rho_{1}\right)} \lim _{T \rightarrow \infty}\left(\frac{1}{\#\left(R_{T}(\rho)\right)} \sum_{[\alpha] \in R_{T}\left(\rho_{1}\right)} \frac{\ell_{\rho_{2}}(\alpha)}{\ell_{\rho_{1}}(\alpha)}\right) .
$$


Bridgeman and Taylor [7] showed that the Hessian of $J$ gives rise to a non-negative bilinear form on $T Q F(S)$, called the pressure form. Motivated by work of McMullen [24] in the setting of Teichmüller space, Bridgeman [5] used the thermodynamic formalism to show that the only degenerate vectors for the pressure form correspond to pure bending at points on the Fuchsian locus. Moreover, the pressure form gives rise to a path metric on $Q F(S)$, called the pressure metric (see also [6, Cor. 1.7]).

We say a smooth immersion $f: Q F(S) \rightarrow Q F(S)$ is a smooth isometry of the renormalized intersection number if

$$
J\left(f\left(\rho_{1}\right), f\left(\rho_{2}\right)\right)=J\left(\rho_{1}, \rho_{2}\right)
$$

for all $\rho_{1}, \rho_{2} \in Q F(S)$. We recall that the (extended) mapping class group $\operatorname{Mod}^{*}(S)$ is the group of isotopy classes of homeomorphisms of $S$. Since $J$ is invariant under the action of $\operatorname{Mod}^{*}(S)$, every element of $\operatorname{Mod}^{*}(S)$ is a smooth isometry of the renormalized intersection number. There exists an involution $\tau: Q F(S) \rightarrow Q F(S)$ given by taking $[\rho]$ to $[\bar{\rho}]$. Since $\tau$ preserves the marked length spectrum, it is an isometry of the renormalized intersection number. We use our main result and work of Bonahon [4] to show that these give rise to all smooth isometries of the renormalized intersection number.

Theorem 1.4. If $S$ is a closed, orientable surface of genus at least two, then the group of smooth isometries of the renormalized intersection number on $Q F(S)$ is generated by the (extended) mapping class group $\operatorname{Mod}^{*}(S)$ and complex conjugation $\tau$.

In the proof of Theorem 1.4, we establish the following strengthening of our main result which may be of independent interest.

Theorem 1.5. If $S$ is a closed, connected, orientable surface of genus at least two, $\rho_{1}: \pi_{1}(S) \rightarrow \operatorname{PSL}(2, \mathbb{C})$ and $\rho_{2}: \pi_{1}(S) \rightarrow \operatorname{PSL}(2, \mathbb{C})$ are Kleinian surface groups, and there exists $k$ so that and $\ell_{\rho_{1}}(\alpha)=k \ell_{\rho_{2}}(\alpha)$ for all $\alpha \in \pi_{1}(S)$ which are represented by simple curves on $S$, then $\rho_{1}$ is conjugate to either $\rho_{2}$ or $\bar{\rho}_{2}$.

Historical Remarks: Royden [29] showed that $\operatorname{Mod}^{*}(S)$ is the isometry group of the Teichmüller metric on $T(S)$. Masur and Wolf [23] proved that $\operatorname{Mod}^{*}(S)$ is the isometry group of the Weil-Petersson metric on $T(S)$. Bridgeman [5] used work of Wolpert [33] to show that the restriction of the pressure form to the Fuchsian locus is a multiple of the Weil-Petersson metric.

One may thus view Theorem 1.4 as evidence in favor of the following natural conjecture.

Conjecture: The isometry group of the pressure metric on quasifuchsian space $Q F(S)$ is generated by the (extended) mapping class group and complex conjugation.

Kim [18, Thm. 3] showed that if $\rho_{1}$ and $\rho_{2}$ are irreducible, non-elementary, nonparabolic representations of a finitely presented group $\Gamma$ into the isometry group of a rank one symmetric space and there exists $k>0$ such that $\ell_{\rho_{1}}(\gamma)=k \ell_{\rho_{2}}(\gamma)$ for all $\gamma \in \Gamma$ (where $\ell_{\rho_{i}}(\gamma)$ ) the translation length of $\left.\rho_{i}(\gamma)\right)$, then $k=1$ and $\rho_{1}$ and $\rho_{2}$ are conjugate representations.

Outline of paper: In section 2 we analyze the complex length spectrum of Kleinian surface groups with the same simple marked length spectrum. In section 3 , we give the 
proof of our main result, while in section 4 we give the quick proof of Corollary 1.2. In section 5 we prove Theorem 1.3, while in section 6 we establish Theorems 1.4 and 1.5

Acknowledgements: The authors would like to thank Maxime Wolff for several enlightening conversations on the length spectra of surface group representations and Jeff Brock and Mike Wolf for conversations about the Weil-Petersson metric. This material is partially based upon work supported by the National Science Foundation under grant No. 0932078 000 while the authors were in residence at the Mathematical Sciences Research Institute in Berkeley, CA, during the Spring 2015 semester.

\section{The COMPleX LENGTH SPECTRUM}

In this section, we investigate the complex length spectra of Kleinian surface groups with the same simple marked length spectrum.

Given $\alpha \in \pi_{1}(S)$ and $\rho \in A H(S)$, let $\lambda_{\rho}^{2}(\alpha)$ be the square of the largest eigenvalue of $\rho(\alpha)$. Notice that $\lambda_{\rho}^{2}(\alpha)$ is well-defined even though the largest eigenvalue of a matrix in $\operatorname{PSL}(2, \mathbb{C})$ is only well-defined up to sign. If we choose $\log \lambda_{\rho}^{2}(\alpha)$ to have imaginary part in $(-\pi, \pi]$, then $\log \lambda_{\rho}^{2}(\alpha)$ is the complex length of $\rho(\alpha)$.

If $\alpha$ is a simple, non-separating closed curve on $S$, we let $W(\alpha)$ denote the set of all simple, non-separating curves on $S$ which intersect $\alpha$ at most once. We say that $\rho_{1}$ and $\rho_{2}$ have the same marked complex length spectrum on $W(\alpha)$ if $\lambda_{\rho_{1}}^{2}(\beta)=\lambda_{\rho_{2}}^{2}(\beta)$ for all $\beta \in W(\alpha)$. Similarly, we say that $\rho_{1}$ and $\rho_{2}$ have conjugate marked complex length spectrum on $W(\alpha)$ if $\lambda_{\rho_{1}}^{2}(\beta)=\overline{\lambda_{\rho_{2}}^{2}(\beta)}$ for all $\beta \in W(\alpha)$.

We will show that if two Kleinian surface groups $\rho_{1}$ and $\rho_{2}$ have the same simple marked length spectrum, then, there exists a simple non-separating curve $\alpha$ on $S$ such that $\rho_{1}$ and $\rho_{2}$ either have the same or conjugate complex length spectrum on $W(\alpha)$.

Proposition 2.1. If $S$ is a closed, connected, orientable surface of genus at least two, $\rho: \pi_{1}(S) \rightarrow \operatorname{PSL}(2, \mathbb{C})$ and $\rho_{2}: \pi_{1}(S) \rightarrow \operatorname{PSL}(2, \mathbb{C})$ are Kleinian surface groups with the same simple marked length spectrum, then there exists a simple non-separating curve $\alpha$ on $S$ such that $\rho_{1}(\alpha)$ is hyperbolic and either

(1) $\rho_{1}$ and $\rho_{2}$ have the same marked complex length spectrum on $W(\alpha)$, or

(2) $\rho_{1}$ and $\rho_{2}$ have conjugate marked complex length spectrum on $W(\alpha)$.

Proposition 2.1 will be a nearly immediate consequence of three lemmas. The first lemma shows that for two Kleinian surface groups with the same length spectrum, then the complex lengths of a simple non-separating curve either agree, differ by complex conjugation, or differ by sign (and are both real). The second lemma deals with the case where the complex length of every simple, non-separating curve is real, while the final lemma handles the case where some complex length is not real. All the proofs revolve around an analysis of the asymptotic behavior of complex lengths of curves of the form $\alpha^{n} \beta$ where $\alpha$ and $\beta$ intersect exactly once. We begin by recording computations which will be used repeatedly in the remainder of the paper. 
2.1. A convenient normalization. We recall that two elements $\alpha, \beta \in \pi_{1}(S)$ are coprime if they share no common powers. We say that a representation $\rho: \pi_{1}(S) \rightarrow \operatorname{PSL}(2, \mathbb{C})$ is $(\alpha, \beta)$-normalized if $\alpha, \beta \in \pi_{1}(S)$ are coprime and $\rho(\alpha)$ is hyperbolic and has attracting fixed point $\infty$ and repelling fixed point 0 . In this case,

$$
\rho(\alpha)= \pm\left(\begin{array}{cc}
\lambda & 0 \\
0 & \lambda^{-1}
\end{array}\right)
$$

where $|\lambda|>1$, and

$$
\rho(\beta)= \pm\left(\begin{array}{ll}
a & b \\
c & d
\end{array}\right)
$$

where $a d-b c=1$. Notice that the matrix representations of elements of $\operatorname{PSL}(2, \mathbb{C})$ are only well-defined up to multiplication by $\pm I$, but many related quantities like the square of the trace, the product of any two co-efficients, and the modulus of the eigenvalue of maximal modulus are well-defined.

Lemma 2.2. Suppose that $S$ is a closed, connected, orientable surface of genus at least two and $\rho: \pi_{1}(S) \rightarrow \operatorname{PSL}(2, \mathbb{C})$ is an $(\alpha, \beta)$-normalized Kleinian surface group. In the above notation,

$$
\rho\left(\alpha^{n} \beta\right)= \pm\left(\begin{array}{cc}
\lambda^{n} a & \lambda^{n} b \\
\lambda^{-n} c & \lambda^{-n} d
\end{array}\right)
$$

and all the matrix coefficients of $\rho(\beta)$ are non-zero. Moreover, if $|\mu(n)|$ is the modulus of the eigenvalue of $\rho\left(\alpha^{n} \beta\right)$ with largest modulus, then

$$
\log |\mu(n)|=n \log |\lambda|+\log |a|+\Re\left(\lambda^{-2 n} \frac{b c}{a^{2}}\right)+O\left(|\lambda|^{-4 n}\right) .
$$

Proof. The first claim follows from a simple computation. If any of the coefficients of $\rho(\beta)$ are 0 , then $\rho(\beta)$ takes some fixed point of $\rho(\alpha)$ to a fixed point of $\rho(\alpha)$, e.g. if $a=0$, then $\rho(\beta)(\infty)=0$. This would imply that $\rho\left(\beta \alpha \beta^{-1}\right)$ shares a fixed point with $\rho(\alpha)$. Since $\rho\left(\pi_{1}(S)\right)$ is discrete, this would imply that there is an element which is a power of both $\rho(\alpha)$ and $\rho\left(\beta \alpha \beta^{-1}\right)$ which would contradict the facts that $\rho$ is faithful and the subgroup of $\pi_{1}(S)$ generated by $\alpha$ and $\beta$ is free of rank two.

Solving for $\mu(n)$, we see that

$$
\mu(n)= \pm\left(\frac{\left(\lambda^{n} a+\lambda^{-n} d\right) \pm \sqrt{\left(\lambda^{n} a+\lambda^{-n} d\right)^{2}-4}}{2}\right)
$$

So, since $|\lambda|>1$, for all large enough $n$, one may use the Taylor expansion for $\sqrt{1+x}$ to conclude that

$$
\mu(n)= \pm\left(\lambda^{n} a\left(1+\lambda^{-2 n}\left(\frac{a d-1}{a^{2}}\right)+O\left(\lambda^{-4 n}\right)\right)\right)
$$

Therefore, since $a d-b c=1$,

$$
\log |\mu(n)|=n \log |\lambda|+\log |a|+\log \left|1+\lambda^{-2 n} \frac{b c}{a^{2}}+O\left(\lambda^{-4 n}\right)\right| .
$$


We then use the expansion of $\log |1+z|$ about $z=0$ given by

$$
\log |1+z|=\frac{1}{2} \log \left(|1+z|^{2}\right)=\frac{1}{2} \log \left(1+2 \Re(z)+|z|^{2}\right)=\Re(z)+O\left(|z|^{2}\right)
$$

to show that

$$
\log |\mu(n)|=n \log |\lambda|+\log |a|+\Re\left(\lambda^{-2 n} \frac{b c}{a^{2}}\right)+O\left(|\lambda|^{-4 n}\right) .
$$

2.2. Basic relationships between complex lengths. Our first lemma shows that if two Kleinian surface groups have the same simple marked length spectrum, then the complex lengths of any simple, non-separating curve either agree or differ by either complex conjugation or sign.

Lemma 2.3. If $\rho_{1}$ and $\rho_{2}$ in $A H(S)$ have the same simple marked length spectrum and $\alpha$ is a simple non-separating curve on $S$, then either

(1) $\lambda_{\rho_{1}}^{2}(\alpha)=\lambda_{\rho_{2}}^{2}(\alpha)$,

(2) $\lambda_{\rho_{1}}^{2}(\alpha)=\overline{\lambda_{\rho_{2}}^{2}(\alpha)}$, or

(3) $\lambda_{\rho_{1}}^{2}(\alpha)=-\lambda_{\rho_{2}}^{2}(\alpha)$ and $\lambda_{\rho_{1}}^{2}(\alpha)$ is real.

Proof. If $\rho_{1}(\alpha)$ is parabolic, then $\rho_{2}(\alpha)$ is parabolic (since $\left.\ell_{\rho_{2}}(\alpha)=\ell_{\rho_{1}}(\alpha)=0\right)$. In this case, $\lambda_{\rho_{1}}^{2}(\alpha)=\lambda_{\rho_{2}}^{2}(\alpha)=1$. So we may assume that $\rho(\alpha)$ is hyperbolic.

Let $\beta$ be a curve intersecting $\alpha$ exactly once. We may assume that both $\rho_{1}$ and $\rho_{2}$ are $(\alpha, \beta)$-normalized, so

$$
\rho_{i}(\alpha)= \pm\left(\begin{array}{cc}
\lambda_{i} & 0 \\
0 & \lambda_{i}^{-1}
\end{array}\right)
$$

where $\left|\lambda_{i}\right|>1$, and

$$
\rho_{i}(\beta)= \pm\left(\begin{array}{cc}
a_{i} & b_{i} \\
c_{i} & d_{i}
\end{array}\right)
$$

where $a_{i} d_{i}-b_{i} c_{i}=1$.

Since, $\rho_{1}$ and $\rho_{2}$ have the same simple marked length spectrum and $\left|\lambda_{1}\right|=\left|\lambda_{2}\right|$, Lemma 2.2 implies that

$$
\log \left|a_{1}\right|+\Re\left(\lambda_{1}^{-2 n} \frac{b_{1} c_{1}}{a_{1}^{2}}\right)+O\left(\left|\lambda_{1}\right|^{-4 n}\right)=\log \left|a_{2}\right|+\Re\left(\lambda_{2}^{-2 n} \frac{b_{2} c_{2}}{a_{2}^{2}}\right)+O\left(\left|\lambda_{2}\right|^{-4 n}\right)
$$

for all $n$. Taking limits as $n \rightarrow \infty$, we see that $\log \left|a_{1}\right|=\log \left|a_{2}\right|$, so

$$
\Re\left(\lambda_{1}^{-2 n} \frac{b_{1} c_{1}}{a_{1}^{2}}\right)+O\left(\left|\lambda_{1}\right|^{-4 n}\right)=\Re\left(\lambda_{2}^{-2 n} \frac{b_{2} c_{2}}{a_{2}^{2}}\right)+O\left(\left|\lambda_{2}\right|^{-4 n}\right)
$$

for all $n$. Therefore, after multiplying both sides by $\left|\lambda_{1}\right|^{2 n}=\left|\lambda_{2}\right|^{2 n}$, we see that

$$
\lim _{n \rightarrow \infty} \Re\left(u_{1}^{n} v_{1}-u_{2}^{n} v_{2}\right)=0
$$


where

$$
u_{i}=\left(\frac{\lambda_{i}}{\left|\lambda_{i}\right|}\right)^{-2} \text { and } \quad v_{i}=\frac{b_{i} c_{i}}{a_{i}^{2}} \neq 0 .
$$

Lemma 2.3 is then an immediate consequence of the following elementary lemma.

Lemma 2.4. If $u_{1}, u_{2} \in \mathbb{S}^{1}, v_{1}, v_{2} \in \mathbb{C}-\{0\}$ and

$$
\lim _{n \rightarrow \infty} \Re\left(u_{1}^{n} v_{1}-u_{2}^{n} v_{2}\right)=0,
$$

then either

(1) $u_{1}=u_{2}$,

(2) $u_{1}=\bar{u}_{2}$, or

(3) $u_{1}=-u_{2}= \pm 1$.

Proof. We choose $\theta_{i}$ so that

$$
u_{i}=e^{i \theta_{i}}
$$

If $s=\left\{n_{k}\right\}_{k=1}^{\infty}$ is a strictly increasing sequence of integers, let $S_{i}(s)$ be the set of accumulation points of $\left\{\Re\left(u_{i}^{n_{k}} v_{i}\right)\right\}$. Then, by assumption, $S_{1}(s)=S_{2}(s)$ for any sequence $s$.

If $\theta_{i}$ is an irrational multiple of $2 \pi$, then $S_{i}(\mathbb{N})$ is the interval $\left[-\left|v_{i}\right|,\left|v_{i}\right|\right]$. If $\theta_{i}$ is a rational multiple of $2 \pi$ then $S_{i}(\mathbb{N})$ is finite. Therefore either (a) both $\theta_{1}$ and $\theta_{2}$ are irrational with $\left|v_{1}=\right| v_{2} \mid$ or (b) both $\theta_{1}$ and $\theta_{2}$ are rational multiples of $2 \pi$. We handle these two cases separately.

Case (a): Both $\theta_{1}$ and $\theta_{2}$ are irrational multiples of $2 \pi$ and $\left|v_{1}\right|=\left|v_{2}\right|$ : Since $\theta_{1}$ is an irrational multiple of $2 \pi$, there is a sequence $\left\{n_{k}\right\}$ such that $\lim _{k \rightarrow \infty} e^{i n_{k} \theta_{1}}=\frac{\overline{v_{1}}}{\left|v_{1}\right|}$. Therefore,

$$
\left|v_{1}\right|=\lim _{k \rightarrow \infty} \Re\left(e^{i n_{k} \theta_{1}} v_{1}\right)=\lim _{k \rightarrow \infty} \Re\left(e^{i n_{k} \theta_{2}} v_{2}\right)=\left|v_{2}\right|,
$$

so $\lim _{k \rightarrow \infty} e^{i n_{k} \theta_{2}}=\frac{\bar{v}_{2}}{\left|v_{2}\right|}$. If $\left\{m_{k}\right\}=\left\{n_{k}+1\right\}$, then

$$
\left|v_{1}\right| \cos \theta_{1}=\lim _{k \rightarrow \infty} \Re\left(e^{i m_{k} \theta_{1}} v_{1}\right)=\lim _{k \rightarrow \infty} \Re\left(e^{i m_{k} \theta_{2}} v_{2}\right)=\left|v_{2}\right| \cos \theta_{2} .
$$

Since $\left|v_{1}\right|=\left|v_{2}\right| \neq 0$, it follows that $\theta_{1}= \pm \theta_{2}$, so either $u_{1}=u_{2}$ or $u_{1}=\bar{u}_{2}$ and we are either in case (1) or in case (2).

Case II: Both $\theta_{1}$ and $\theta_{2}$ are rational multiples of $2 \pi$ : Let $\theta_{i}=2 \pi p_{i} / q_{i}$ where $0 \leq p_{i}<q_{i}$ and $p_{1}$ and $q_{i}$ are relatively prime (and $q_{i}=1$ if $p_{i}=0$ ).

If $r \in \mathbb{Z}$ and $s_{r}=\left\{r+k q_{1} q_{2}\right\}$, then

$$
S_{1}\left(s_{r}\right)=\left\{\Re\left(u_{1}^{r} v_{1}\right)\right\}=S_{2}\left(s_{r}\right)=\left\{\Re\left(u_{2}^{r} v_{2}\right)\right\},
$$

so

$$
\Re\left(u_{1}^{r} v_{1}\right)=\Re\left(u_{2}^{r} v_{2}\right) \quad \text { for all } r \in \mathbb{Z} .
$$

If $\Re\left(u_{1}^{r} v_{1}\right)=\Re\left(u_{2}^{r} v_{2}\right)=0$ for all $r \in \mathbb{Z}$, then $u_{1}= \pm u_{2}= \pm 1$, and we are in either case (1) or case (3). 
If $\Re\left(u_{1}^{r} v_{1}\right)=\Re\left(u_{2}^{r} v_{2}\right) \neq 0$ for some $r$, then

$$
2 \cos \left(\theta_{1}\right) \Re\left(u_{1}^{r} v_{1}\right)=\Re\left(u_{1}^{r+1} v_{1}\right)+\Re\left(u_{1}^{r-1} v_{1}\right)=\Re\left(u_{2}^{r+1} v_{2}\right)+\Re\left(u_{2}^{r-1} v_{2}\right)=2 \cos \left(\theta_{2}\right) \Re\left(u_{2}^{r} v_{2}\right) .
$$

Since $\Re\left(u_{1}^{r} v_{1}\right)=\Re\left(u_{2}^{r} v_{2}\right) \neq 0$, this implies that $\cos \left(\theta_{1}\right)=\cos \left(\theta_{2}\right)$, so $\theta_{1}= \pm \theta_{2}$. Therefore, either $u_{1}=u_{2}$ or $u_{1}=\bar{u}_{2}$ and we are in either case (1) or case (2).

This completes the proof, since in all situations we have seen that either case (1), (2) or (3) occurs.

2.3. When the simple non-separating complex length spectrum is totally real. We use a similar analysis to show that if the complex lengths of every simple, non-separating curve is real for two Kleinian surface groups with the same simple marked length spectrum, then the complex lengths agree for every simple, non-separating curve.

Lemma 2.5. If $S$ is a closed, connected, orientable surface of genus at least two and $\rho_{1}: \pi_{1}(S) \rightarrow \operatorname{PSL}(2, \mathbb{C})$ and $\rho_{2}: \pi_{1}(S) \rightarrow \operatorname{PSL}(2, \mathbb{C})$ are Kleinian surface groups with same simple marked length spectrum, then either

(1) there exists a simple non-separating curve $\gamma$ on $S$ such that $\lambda_{\rho_{1}}^{2}(\gamma) \notin \mathbb{R}$, or

(2) $\lambda_{\rho_{1}}^{2}(\gamma)=\lambda_{\rho_{2}}^{2}(\gamma) \in \mathbb{R}$ whenever $\gamma$ is a simple non-separating curve on $S$.

Proof. Suppose that 1) does not hold, so $\lambda_{\rho_{1}}^{2}(\gamma) \in \mathbb{R}$ whenever $\gamma$ is a simple non-separating curve on $S$. Lemma 2.3 then implies that $\lambda_{\rho_{1}}^{2}(\gamma)= \pm \lambda_{\rho_{2}}^{2}(\gamma)$ whenever $\gamma$ is a simple nonseparating curve on $S$

Suppose that there is a simple non-separating curve $\alpha$ such that $\lambda_{\rho_{1}}^{2}(\alpha)=-\lambda_{\rho_{2}}^{2}(\alpha)$. Notice that if $\rho_{1}(\alpha)$ is parabolic, then $\ell_{\rho_{1}}(\alpha)=0=\ell_{\rho_{2}}(\alpha)$, so $\lambda_{\rho_{1}}^{2}(\alpha)=1=\lambda_{\rho_{2}}^{2}(\alpha)$. Therefore, $\rho_{1}(\alpha)$, and hence $\rho_{2}(\alpha)$, must be hyperbolic

We choose a simple non-separating curve $\beta$ intersecting $\alpha$ exactly once. We adapt the normalization and notation of Lemma 2.3. Lemma 2.2 implies that

$$
t_{i}(n)=\operatorname{Tr}^{2}\left(\rho_{i}\left(\alpha^{n} \beta\right)\right)=\lambda_{i}^{2 n} a_{i}^{2}+2 a_{i} d_{i}+\lambda_{i}^{-2 n} d_{i}^{2}=\lambda_{\rho_{i}}^{2}\left(\alpha^{n} \beta\right)+2+\lambda_{\rho_{i}}^{-2}\left(\alpha^{n} \beta\right) .
$$

(Notice that the trace $\operatorname{Tr}\left(\rho_{i}\left(\alpha^{n} \beta\right)\right)$ of $\rho_{i}\left(\alpha^{n} \beta\right)$ is well-defined up to sign, so $\operatorname{Tr}^{2}\left(\rho_{i}\left(\alpha^{n} \beta\right)\right)$ is well-defined.) Since $\lambda_{\rho_{1}}^{2}\left(\alpha^{n} \beta\right)= \pm \lambda_{\rho_{2}}^{2}\left(\alpha^{n} \beta\right)$ for all $n$, by assumption, either (i) $t_{1}(n)=t_{2}(n)$ or (ii) $t_{1}(n)=4-t_{2}(n)$ for all $n$ and $\lambda_{1}^{2}=-\lambda_{2}^{2}$.

The proof divides into two cases.

Case I: There is an infinite sequence $\left\{n_{k}\right\}$ of even integers so that $t_{1}\left(n_{k}\right)=t_{2}\left(n_{k}\right)$ : Dividing by $\lambda_{1}^{2 n_{k}}=\lambda_{2}^{2 n_{k}}$ and taking limits we see that

$$
a_{1}^{2}=\lim _{k \rightarrow \infty} a_{1}^{2}+\frac{2 a_{1} d_{1}}{\lambda_{1}^{2 n_{k}}}+\frac{d_{1}^{2}}{\lambda_{1}^{4 n_{k}}}=\lim _{k \rightarrow \infty} a_{2}^{2}+\frac{2 a_{2} d_{2}}{\lambda_{2}^{2 n_{k}}}+\frac{d_{2}^{2}}{\lambda_{2}^{4 n_{k}}}=a_{2}^{2} .
$$

It follows that

$$
2 a_{1} d_{1}+\frac{d_{1}^{2}}{\lambda_{1}^{2 n_{k}}}=2 a_{2} d_{2}+\frac{d_{2}^{2}}{\lambda_{2}^{2 n_{k}}}
$$

for all $n_{k}$, so, after again taking limits, we see that

$$
a_{1} d_{1}=a_{2} d_{2} \text {. }
$$


Looking at the final term, we see that

$$
d_{1}^{2}=d_{2}^{2}
$$

If there exists an infinite sequence $\left\{m_{j}\right\}$ of odd integers so that $t_{1}\left(m_{j}\right)=t_{2}\left(m_{j}\right)$ for all $m_{j}$, then

$$
\lambda_{1}^{2 m_{j}} a_{1}^{2}+2 a_{1} d_{1}+\lambda_{1}^{-2 m_{j}} d_{1}^{2}=-\lambda_{1}^{2 m_{j}} a_{1}^{2}+2 a_{1} d_{1}-\lambda_{1}^{-2 m_{j}} d_{1}^{2} .
$$

Then we may divide each side by $\lambda_{1}^{2 m_{j}}$ and pass to a limit to conclude that $a_{1}^{2}=-a_{1}^{2}$. This would imply that $a_{1}=0$, which would contradict Lemma 2.2 .

On the other hand, if there exists an infinite sequence $\left\{m_{j}\right\}$ of odd integers so that $t_{1}\left(m_{j}\right)=4-t_{2}\left(m_{j}\right)$ for all $m_{j}$, then

$$
\lambda_{1}^{2 m_{j}} a_{1}^{2}+2 a_{1} d_{1}+\lambda_{1}^{-2 m_{j}} d_{1}^{2}=4-\left(-\lambda_{1}^{2 m_{j}} a_{1}^{2}+2 a_{1} d_{1}-\lambda_{1}^{-2 m_{j}} d_{1}^{2}\right),
$$

so

$$
2 a_{1} d_{1}=4-2 a_{1} d \text {. }
$$

Therefore, $a_{1} d_{1}=1$, which implies that $b_{1} c_{1}=0$, so either $b_{1}=0$ or $c_{1}=0$, which again contradicts Lemma 2.2.

Case II: There is an infinite sequence $\left\{n_{k}\right\}$ of even integers so that $t_{1}\left(n_{k}\right)=4-t_{2}\left(n_{k}\right)$ : We then argue, as in Case I, to show that

$$
a_{1}^{2}=-a_{2}^{2} \quad \text { and } \quad 2 a_{1} d_{1}=4-2 a_{2} d_{2} \quad \text { and } \quad d_{1}^{2}=-d_{2}^{2}
$$

If there exists an infinite sequence $\left\{m_{j}\right\}$ of odd integers so that $t_{1}\left(m_{j}\right)=t_{2}\left(m_{j}\right)$ for all $m_{j}$, then

$$
\lambda_{1}^{2 m_{j}} a_{1}^{2}+2 a_{1} d_{1}+\lambda_{1}^{-2 m_{j}} d_{1}^{2}=\lambda_{1}^{2 m_{j}} a_{1}^{2}+\left(4-2 a_{1} d_{1}\right)+\lambda_{1}^{-2 m_{j}} d_{1}^{2} .
$$

So, $a_{1} d_{1}=1$, again giving a contradiction.

On the other hand, if there exists an infinite sequence $\left\{m_{j}\right\}$ of odd integers so that $t_{1}\left(m_{j}\right)=4-t_{2}\left(m_{j}\right)$ for all $m_{j}$, then

$$
\lambda_{1}^{2 m_{j}} a_{1}^{2}+2 a_{1} d_{1}+\lambda_{1}^{-2 m_{j}} d_{1}^{2}=4-\left(\lambda_{1}^{2 m_{j}} a_{1}^{2}+\left(4-2 a_{1} d_{1}\right)+\lambda_{1}^{-2 m_{j}} d_{1}^{2}\right) .
$$

Dividing both sides by $\lambda_{1}^{2 m_{j}}$ and passing to a limit, we conclude that $a_{1}^{2}=-a_{1}^{2}$, which is again a contradiction.

Therefore, neither Case I or Case II can occur, so case (2) must hold.

2.4. When the complex length is not always totally real. We now show that if $\lambda_{\rho_{1}}^{2}(\alpha)$ is not real, for some simple non-separating curve $\alpha$, then $\lambda_{\rho_{1}}^{2}(\beta)$ and $\lambda_{\rho_{2}}^{2}(\beta)$ either agree for all $\beta \in W(\alpha)$, or differ by complex conjugation for all $\beta \in W(\alpha)$.

Lemma 2.6. Suppose that $S$ is a closed, connected, orientable surface of genus at least two and $\rho_{1}: \pi_{1}(S) \rightarrow \operatorname{PSL}(2, \mathbb{C})$ and $\rho_{2}: \pi_{1}(S) \rightarrow \operatorname{PSL}(2, \mathbb{C})$ are Kleinian surface groups with same simple marked length spectrum. If $\alpha$ is simple non-separating curve on $S$ such that $\lambda_{\rho_{1}}^{2}(\alpha) \notin \mathbb{R}$, then either

(1) $\rho_{1}$ and $\rho_{2}$ have the same marked complex length spectrum on $W(\alpha)$, or

(2) $\rho_{1}$ and $\rho_{2}$ have conjugate marked complex length spectrum on $W(\alpha)$. 
Proof. Lemma 2.3 implies that either $\lambda_{\rho_{1}}^{2}(\alpha)=\lambda_{\rho_{2}}^{2}(\alpha)$ or $\lambda_{\rho_{1}}^{2}(\alpha)=\overline{\lambda_{\rho_{2}}^{2}(\alpha)}$. If $\lambda_{\rho_{1}}^{2}(\alpha)=$ $\overline{\lambda_{\rho_{2}}^{2}(\alpha)}$, then we consider the representation $\bar{\rho}_{2}$. In this case, $\lambda_{\rho_{2}}^{2}(\gamma)=\overline{\lambda_{\bar{\rho}_{2}}^{2}(\gamma)}$ for all $\gamma \in \pi_{1}(S)$. In particular, $\lambda_{\rho_{1}}^{2}(\alpha)=\lambda_{\rho_{2}}^{2}(\alpha)$. Therefore, it suffices to prove that $\rho_{1}$ and $\rho_{2}$ have the same marked complex length spectrum on $W(\alpha)$ whenever $\lambda^{2}=\lambda_{\rho_{1}}^{2}(\alpha)=\lambda_{\rho_{2}}^{2}(\alpha)$ and $\rho_{1}$ and $\rho_{2}$ have the same simple marked length spectrum.

First, suppose that $\beta$ is a simple non-separating curve on $S$ which intersects $\alpha$ once. We adopt the normalization and notation of Lemmas 2.3 and 2.5, so

$$
t_{i}(n)=\operatorname{Tr}^{2}\left(\rho_{i}\left(\alpha^{n} \beta\right)\right)=\lambda^{2 n} a_{i}^{2}+2 a_{i} d_{i}+\lambda^{-2 n} d_{i}^{2}
$$

Lemma 2.3 implies that for any $n$, either (i) $t_{1}(n)=t_{2}(n)$, (ii) $t_{1}(n)=\overline{t_{2}(n)}$ or (iii) $t_{1}(n)=4-t_{2}(n)$ and $t_{1}(n)$ is real.

If there is an infinite set of values of $n$ such that $t_{1}(n)=t_{2}(n)$, then, by taking limits, we see that

$$
a_{1}^{2}=a_{2}^{2}, \quad a_{1} d_{1}=a_{2} d_{2} \quad \text { and } \quad d_{1}^{2}=d_{2}^{2} .
$$

It follows then that $t_{1}(n)=t_{2}(n)$ for all $n$. Moreover, since $a_{i}$ and $d_{i}$ are non-zero, either $a_{1}=a_{2}$ and $d_{1}=d_{2}$ or $a_{1}=-a_{2}$ and $d_{1}=-d_{2}$, so $\operatorname{Tr}^{2}\left(\rho_{1}(\beta)\right)=\operatorname{Tr}^{2}\left(\rho_{2}(\beta)\right)$ which implies that $\lambda_{\rho_{1}}^{2}(\beta)=\lambda_{\rho_{2}}^{2}(\beta)$.

If there is an infinite set of values $n$ such that $t_{1}(n)=4-t_{2}(n)$ with $t_{i}(n)$ real, then taking limits we have

$$
a_{1}^{2}=-a_{2}^{2}, \quad 2 a_{1} d_{1}=4-2 a_{2} d_{2} \quad \text { and } \quad d_{1}^{2}=-d_{2}^{2} .
$$

It follows that $t_{1}(n)=4-t_{2}(n)$ for all $n$, so by Lemma $2.3, t_{1}(n)$ is real for all $n$. Since $\Im\left(t_{i}(n)\right)=0$ for all $n$,

$$
\lim _{n \rightarrow \infty} \frac{\Im\left(t_{i}(n)\right)}{|\lambda|^{2 n}}=\lim _{n \rightarrow \infty} \Im\left(\left(\frac{\lambda^{2}}{|\lambda|^{2}}\right)^{n} a_{i}^{2}\right)=0 .
$$

Since $a_{i}^{2} \neq 0$, by Lemma 2.2, this can only happen if $\frac{\lambda^{2}}{|\lambda|^{2}}= \pm 1$. Thus, $\lambda^{2}=\lambda_{\rho_{1}}\left(\alpha^{2}\right)$ is real, contradicting our assumption.

Finally, if $t_{1}(n)=\overline{t_{2}(n)}$ for all but finitely many values of $n$, we may divide the resulting equation by $\overline{\lambda^{2 n}}$ and take a limit, to see that

$$
\lim _{n \rightarrow \infty}\left(\frac{\lambda^{2}}{\overline{\lambda^{2}}}\right)^{n} a_{1}^{2}=\overline{a_{2}^{2}} .
$$

Since $\lambda^{2} \neq \overline{\lambda^{2}}$, the limit does not exist unless $a_{1}=0$, which again contradicts Lemma 2.2.

Therefore, if $\beta \in W(\alpha)$ intersects $\alpha$ once, then $\lambda_{\rho_{1}}^{2}(\beta)=\lambda_{\rho_{2}}^{2}(\beta)$.

Now suppose that $\beta$ is a simple non-separating curve on $S$ which does not intersect $\alpha$. We choose $\beta^{\prime}$ to be a simple non-separating curve intersecting both $\alpha$ and $\beta$ once.

For all $n, \alpha^{n} \beta^{\prime} \in W(\alpha)$ and intersects $\alpha$ once. By the first part of the argument,

$$
\lambda_{\rho_{1}}^{2}\left(\alpha^{n} \beta^{\prime}\right)=\lambda_{\rho_{2}}^{2}\left(\alpha^{n} \beta^{\prime}\right)
$$


for all $n$. If there exists $n_{0}$ so that $\lambda_{\rho_{1}}^{2}\left(\alpha^{n_{0}} \beta^{\prime}\right)$ is not real, then since $\beta \in W\left(\alpha^{n_{0}} \beta^{\prime}\right)$ and intersects $\alpha^{n_{0}} \beta^{\prime}$ exactly once, we may apply the above argument to show that $\lambda_{\rho_{1}}^{2}(\beta)=$ $\lambda_{\rho_{2}}^{2}(\beta)$.

It remains to consider the case that $\lambda_{\rho_{1}}^{2}\left(\alpha^{n} \beta^{\prime}\right)$ is real for all $n$. Suppose that

$$
\rho_{i}\left(\beta^{\prime}\right)= \pm\left(\begin{array}{cc}
a_{i}^{\prime} & b_{i}^{\prime} \\
c_{i}^{\prime} & d_{i}^{\prime}
\end{array}\right) .
$$

Again, by Lemma 2.2 all the matrix coefficients must be non-zero. Since $\Im\left(\lambda_{\rho_{1}}^{2}\left(\alpha^{n} \beta^{\prime}\right)\right)=0$ for all $n, \Im\left(\operatorname{Tr}^{2}\left(\rho_{1}\left(\alpha^{n} \beta^{\prime}\right)\right)\right)=0$ for all $n$, so, after dividing the resulting equation by $|\lambda|^{2 n}$, for all $n$, and passing to the limit we see that

$$
\lim _{n \rightarrow \infty} \Im\left(\left(\frac{\lambda^{2}}{|\lambda|^{2}}\right)^{n} a_{1}^{\prime 2}\right)=0
$$

Since $\frac{\lambda^{2}}{|\lambda|^{2}} \notin \mathbb{R}$, this implies that $a_{1}^{\prime}=0$, which is again a contradiction. Therefore, if $\beta \in W(\alpha)$ does not intersect $\alpha$, then $\lambda_{\rho_{1}}^{2}(\beta)=\lambda_{\rho_{2}}^{2}(\beta)$ which completes the proof.

2.5. Assembly. We can now easily assemble the proof of Proposition 2.1.

Proof of Proposition 2.1: If there exists a simple, non-separating curve $\alpha$ on $S$ so that $\lambda_{\rho_{1}}^{2}(\alpha)$ is not real, then Proposition 2.1 follows immediately from Lemma 2.6. If $\lambda_{\rho_{1}}^{2}(\alpha)$ is real for every simple, non-separating curve $\alpha$ on $S$, then Lemma 2.5 implies that $\rho_{1}$ and $\rho_{2}$ have the same marked complex length spectrum on $W(\alpha)$ for any non-separating simple closed curve $\alpha$. A result of Sullivan [30] implies that there are only finitely many simple curves $\gamma$ on $S$ so that $\rho_{1}(\gamma)$ is parabolic, so we may always choose $\alpha$ so that $\rho_{1}(\alpha)$ is hyperbolic.

Remark: An examination of the proofs reveals that Proposition 2.1 holds whenever the length spectra of $\rho_{1}$ and $\rho_{2}$ agree on all simple, non-separating curves.

\section{Simple Marked Length Spectrum Rigidity}

We are now ready for the proof of our main result.

Theorem 1.1: (Simple length rigidity for Kleinian surface groups) If $S$ is a closed, connected, orientable surface of genus at least two, and $\rho_{1}: \pi_{1}(S) \rightarrow \operatorname{PSL}(2, \mathbb{C})$ and $\rho_{2}: \pi_{1}(S) \rightarrow \operatorname{PSL}(2, \mathbb{C})$ are Kleinian surface groups with the same simple marked length spectrum, then $\rho_{1}$ is conjugate to either $\rho_{2}$ or $\bar{\rho}_{2}$.

We begin with a brief sketch of the proof. It follows from Lemma 2.1 that, perhaps after replacing $\rho_{2}$ with a complex conjugate representation, there exists a simple, non-separating curve $\alpha$ so that $\rho_{1}(\alpha)$ is hyperbolic and $\rho_{1}$ and $\rho_{2}$ have the same marked complex length spectrum on $W(\alpha)$. We then lift $\rho_{1}$ and $\rho_{2}$ to representations into $\operatorname{SL}(2, \mathbb{C})$ which have the same trace on a standard set of generators $\left\{\alpha_{1}, \beta_{1}, \ldots, \alpha_{g}, \beta_{g}\right\}$ where $\alpha=\alpha_{1}$, see Lemma 3.1. An analysis of the asymptotic behavior of the traces of $\alpha_{j}^{n} \beta$ allows us to conclude that the restriction of the lifts to any subgroup of the form $G_{j}=<\alpha_{j}, \beta_{j}>$ are conjugate, see Lemma 3.2. A more intricate analysis of the same type is then applied to show that if we 
conjugate the lifts to agree on $G_{j}$, then, for any $k$, they either agree on $G_{k}$ or differ by conjugation by a lift of the rotation of order two in the axis of the image of the commutator of $\left[\alpha_{j}, \beta_{j}\right]$, see Lemmas 3.3 and 3.4. The proof is then easily completed when the genus is greater than two, see Lemma 3.5, but a separate analysis is required when the genus is two, see Lemma 3.6.

Proof of Theorem 1.1: Proposition 2.1 implies that there exists a a simple non-separating curve $\alpha$ such that $\rho_{1}(\alpha)$ is hyperbolic and $\rho_{1}$ and $\rho_{2}$ have the same marked complex length spectrum on $W(\alpha)$. If $\rho_{1}$ and $\rho_{2}$ have conjugate complex marked length spectrum on $W(\alpha)$, then $\rho_{1}$ and $\bar{\rho}_{2}$ have the same marked complex length spectrum on $W(\alpha)$. Therefore, we may assume that $\rho_{1}$ and $\rho_{2}$ have the same marked complex length spectrum on $W(\alpha)$.

We begin by choosing lifts whose traces agree on a standard set of generators which includes $\alpha$. We will call $\mathcal{S}=\left\{\alpha_{1}, \beta_{1}, \ldots, \alpha_{g}, \beta_{g}\right\}$ a standard set of generators for $\pi_{1}(S)$ if each $\alpha_{j}$ and $\beta_{j}$ is non-separating, $\pi_{1}(S)=<\alpha_{j}, \beta_{j} \mid \prod_{j=1}^{g}\left[\alpha_{i}, \beta_{i}\right]=i d>$ and $i\left(\alpha_{j}, \beta_{j}\right)=1$ for all $j$ and if $j \neq k$ then

$$
i\left(\alpha_{j}, \alpha_{k}\right)=i\left(\beta_{j}, \beta_{k}\right)=i\left(\alpha_{j}, \beta_{k}\right)=0,
$$

see figure 1. We say that two lifts $\tilde{\rho}_{1}$ and $\tilde{\rho}_{2}$ of $\rho_{1}$ and $\rho_{2}$ are trace normalized with respect to $\mathcal{S}$ if

(1) $\rho_{1}(\delta)$ is hyperbolic for all $\delta \in \mathcal{S}$, and

(2) $\operatorname{Tr}\left(\tilde{\rho}_{1}(\delta)\right)=\operatorname{Tr}\left(\tilde{\rho}_{2}(\delta)\right)$ for all $\delta \in \mathcal{S}$.

Lemma 3.1. Suppose that $\rho_{1}: \pi_{1}(S) \rightarrow \operatorname{PSL}(2, \mathbb{C})$ and $\rho_{2}: \pi_{1}(S) \rightarrow \operatorname{PSL}(2, \mathbb{C})$ are Kleinian surface groups with the same marked complex length spectrum on $W(\alpha)$ for some simple, non-separating curve $\alpha$. If $\rho_{1}(\alpha)$ is hyperbolic, then there exists a standard set of generators $\mathcal{S}$, so that $\alpha_{1}=\alpha$, and lifts $\tilde{\rho}_{1}: \pi_{1}(S) \rightarrow \mathrm{SL}(2, \mathbb{C})$ and $\tilde{\rho}_{2}: \pi_{1}(S) \rightarrow \mathrm{SL}(2, \mathbb{C})$ of $\rho_{1}$ and $\rho_{2}$ which are trace normalized with respect to $\mathcal{S}$.

Proof. Let $\alpha_{1}=\alpha$. Choose a simple non-separating curve $\beta$ which intersects $\alpha_{1}$ exactly once so that $\rho_{1}\left(\beta_{1}\right)$ is hyperbolic. (We may do so, since, by a result of Sullivan [30], there are only finitely many simple curves $\gamma$ such that $\rho_{1}(\gamma)$ is parabolic and there are infinitely many possibilities for $\beta_{1}$.) Extend $\left\{\alpha_{1}, \beta_{1}\right\}$ to a standard set of generators $\left\{\alpha_{1}, \beta_{1}, \ldots, \alpha_{g}, \beta_{g}\right\}$. We may assume that $\rho_{1}\left(\alpha_{j}\right)$ is hyperbolic for all $j \geq 2$, by replacing $\alpha_{j}$ by $\alpha_{j} \beta_{j}^{n}$ for some $n$ if necessary. We may then assume that $\rho_{1}\left(\beta_{j}\right)$ is hyperbolic for all $j \geq 2$ by replacing $\beta_{j}$ by $\beta_{j} \alpha_{j}^{n}$ for some $n$ if necessary. Notice that $\mathcal{S} \subseteq W(\alpha)$.

Since each $\rho_{i}$ is discrete and faithful, each $\rho_{i}$ lifts to a representation $\rho_{i}^{\prime}: \pi_{1}(S) \rightarrow$ $\operatorname{SL}(2, \mathbb{C})$ (see Culler [12] or Kra [20]). Let

$$
\tilde{\rho}_{2}(\delta)=\left\{\begin{array}{cc}
\rho_{2}^{\prime}(\delta) & \text { if } \operatorname{Tr}\left(\rho_{1}^{\prime}(\delta)\right)=\operatorname{Tr}\left(\rho_{2}^{\prime}(\delta)\right) \\
-\rho_{2}^{\prime}(\delta) & \text { if } \operatorname{Tr}\left(\rho_{1}^{\prime}(\delta)\right)=-\operatorname{Tr}\left(\rho_{2}^{\prime}(\delta)\right)
\end{array}\right.
$$

for all $\delta \in \mathcal{S}$. Notice that $\tilde{\rho}_{i}(\delta)$ is a lift of $\rho_{i}(\delta)$ for all $\delta \in \mathcal{S}$ and that $\tilde{\rho}_{i}\left(\prod_{j=1}^{g}\left[\alpha_{j}, \beta_{j}\right]\right)=I$, since $\rho_{i}^{\prime}\left(\prod_{j=1}^{g}\left[\alpha_{j}, \beta_{j}\right]\right)=I$. Therefore, $\tilde{\rho}_{1}$ and $\tilde{\rho}_{2}$ are lifts of $\rho_{1}$ and $\rho_{2}$ which are trace normalized with respect to $\mathcal{S}$.

We next show that the trace normalized lifts are conjugate on the subgroups $G_{j}=<\alpha_{j}, \beta_{j}>$. 


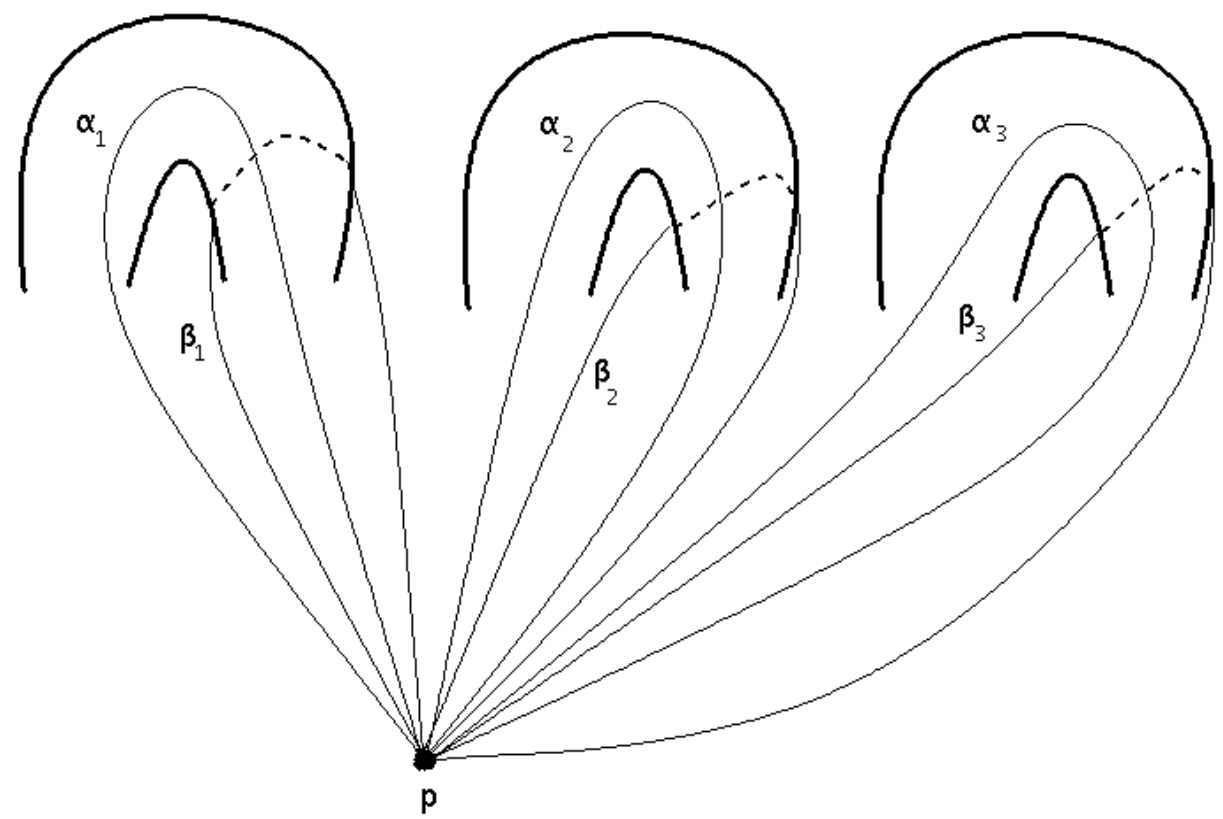

Figure 1. Generators of $\pi_{1}(S, p)$

Lemma 3.2. Suppose that $\tilde{\rho}_{1}: \pi_{1}(S) \rightarrow \mathrm{SL}(2, \mathbb{C})$ and $\tilde{\rho}_{2}: \pi_{1}(S) \rightarrow \mathrm{SL}(2, \mathbb{C})$ are trace normalized lifts, with respect to a standard generating set $\mathcal{S}$, of Kleinian surface groups with the same marked complex length spectrum on $W\left(\alpha_{1}\right)$. If $j \in\{1, \ldots, g\}$, then there exists $K_{j} \in \mathrm{SL}(2, \mathbb{C})$ such that $\left.\tilde{\rho}_{2}\right|_{G_{j}}=\left.\left(K_{j} \tilde{\rho}_{1} K_{j}^{-1}\right)\right|_{G_{j}}$. In particular, if $\gamma \in G_{j}$, then $\operatorname{Tr}\left(\tilde{\rho}_{1}(\gamma)\right)=\operatorname{Tr}\left(\tilde{\rho}_{2}(\gamma)\right)$.

Proof. Fix $j$ for the remainder of the proof of the lemma and assume that $\tilde{\rho}_{1}$ and $\tilde{\rho}_{2}$ are $\left(\alpha_{j}, \beta_{j}\right)$-normalized, so

$$
\tilde{\rho}_{1}\left(\alpha_{j}\right)=\tilde{\rho}_{2}\left(\alpha_{j}\right)=\left(\begin{array}{cc}
\lambda & 0 \\
0 & \lambda^{-1}
\end{array}\right)
$$

where $|\lambda|>1$, and

$$
\tilde{\rho}_{i}\left(\beta_{j}\right)=\left(\begin{array}{cc}
a_{i} & b_{i} \\
c_{i} & d_{i}
\end{array}\right)
$$

where $a_{i} d_{i}-b_{i} c_{i}=1$ and all the co-efficients are non-zero, by Lemma 2.2. Since $\operatorname{Tr}\left(\tilde{\rho}_{1}\left(\beta_{j}\right)\right)=$ $\operatorname{Tr}\left(\tilde{\rho}_{2}\left(\beta_{j}\right)\right)$,

$$
a_{1}+d_{1}=a_{2}+d_{2} .
$$

The curve $\alpha_{j}^{n} \beta_{j} \in W\left(\alpha_{1}\right)$, for all $n$, since it is non-separating, simple and disjoint from $\alpha$ if $j>1$ and intersects $\alpha$ exactly once if $j=1$. Therefore, $\operatorname{Tr}\left(\tilde{\rho}_{1}\left(\alpha_{j}^{n} \beta_{j}\right)\right)= \pm \operatorname{Tr}\left(\tilde{\rho}_{2}\left(\alpha_{j}^{n} \beta_{j}\right)\right)$, which gives the equation

$$
\lambda^{n} a_{1}+\lambda^{-n} d_{1}= \pm\left(\lambda^{n} a_{2}+\lambda^{-n} d_{2}\right)
$$


If $\lambda^{n} a_{1}+\lambda^{-n} d_{1}=-\left(\lambda^{n} a_{2}+\lambda^{-n} d_{2}\right)$ for infinitely many values of $n$, then we see, by dividing by $\lambda^{n}$ and passing to a limit, that $a_{1}=-a_{2}$. It then follows that $d_{1}=-d_{2}$. Thus, $a_{1}+d_{1}=-\left(a_{2}+d_{2}\right)$ which contradicts the fact that the traces of $\tilde{\rho}_{i}$ agree (and are non-zero) on elements of $\mathcal{S}$.

Therefore, there are an infinitely many values of $n$ where $\lambda^{n} a_{1}+\lambda^{-n} d_{1}=\lambda^{n} a_{2}+\lambda^{-n} d_{2}$ Taking limits again, we see that $a_{1}=a_{2}$ and $d_{1}=d_{2}$. Therefore,

$$
b_{1} c_{1}=1-a_{1} d_{1}=1-a_{2} d_{2}=b_{2} c_{2} .
$$

Choose $u \in \mathbb{C}$ such that $u^{2}=b_{2} / b_{1}=c_{1} / c_{2}$. Then $u^{2} b_{1}=b_{2}$ and $u^{-2} c_{1}=c_{2}$. Let

$$
K_{j}=\left(\begin{array}{cc}
u & 0 \\
0 & u^{-1}
\end{array}\right)
$$

Notice that $K_{j} \tilde{\rho}_{1}\left(\alpha_{j}\right) K_{j}^{-1}=\tilde{\rho}_{1}\left(\alpha_{j}\right)$ and that

$$
K_{j} \tilde{\rho}_{1}\left(\beta_{j}\right) K_{j}^{-1}=\left(\begin{array}{cc}
u & 0 \\
0 & u^{-1}
\end{array}\right)\left(\begin{array}{cc}
a_{1} & b_{1} \\
c_{1} & d_{1}
\end{array}\right)\left(\begin{array}{cc}
u^{-1} & 0 \\
0 & u
\end{array}\right)=\left(\begin{array}{cc}
a_{1} & u^{2} b_{1} \\
u^{-2} c_{1} & d_{1}
\end{array}\right)=\tilde{\rho}_{2}\left(\beta_{j}\right) .
$$

Therefore, $K_{j}$ conjugates the restriction of $\tilde{\rho}_{1}$ to $G_{j}$ to the restriction of $\tilde{\rho}_{2}$ to $G_{j}$.

We begin our analysis of trace normalized lifts which agree on $G_{j}$ by examining the relationship between the images of elements of $\mathcal{S}$. Lemma 3.3 is the crucial step in the proof of our main result. The additional information concerning matrix co-efficients in case (2) will only be used when $S$ has genus two.

Lemma 3.3. Suppose that $\rho_{1}^{j}: \pi_{1}(S) \rightarrow \mathrm{SL}(2, \mathbb{C})$ and $\rho_{2}^{j}: \pi_{1}(S) \rightarrow \mathrm{SL}(2, \mathbb{C})$ are trace normalized lifts, with respect to a standard generating set $\mathcal{S}$, of Kleinian surface groups with the same complex marked length spectrum on $W\left(\alpha_{1}\right)$. If $\rho_{1}^{j}$ and $\rho_{2}^{j}$ agree on $G_{j}=<\alpha_{j}, \beta_{j}>$ and $\delta \in \mathcal{S}$, then either

(1) $\rho_{1}^{j}(\delta)=\rho_{2}^{j}(\delta)$, or

(2) $\rho_{1}^{j}\left(\left[\alpha_{j}, \beta_{j}\right]\right)$ is hyperbolic and $R_{j} \rho_{1}^{j}(\delta) R_{j}^{-1}=\rho_{2}^{j}(\delta)$, where $R_{j}$ is a lift of the rotation of order two about the axis of $\rho_{1}^{j}\left(\left[\alpha_{j}, \beta_{j}\right]\right)$.

Moreover, if $\gamma \in G_{j}$ such that $\gamma, \gamma \delta \in W\left(\alpha_{1}\right)$, and

$$
\rho_{1}^{j}\left(\left[\alpha_{j}, \beta_{j}\right]\right)=\left(\begin{array}{cc}
\lambda & 0 \\
0 & \lambda^{-1}
\end{array}\right), \quad \rho_{1}^{j}(\gamma)=\left(\begin{array}{cc}
a & b \\
c & d
\end{array}\right), \quad \text { and } \quad \rho_{1}^{j}(\delta)=\left(\begin{array}{cc}
e & f \\
g & h
\end{array}\right)
$$

where $|\lambda|>1$, then

$$
\frac{e}{h}=-\frac{d}{a}
$$

Proof. The proof breaks up into two cases, depending on whether $\rho_{1}^{j}\left(\left[\alpha_{j}, \beta_{j}\right]\right)$ is hyperbolic or parabolic.

Case I: $\rho_{1}^{j}\left(\left[\alpha_{j}, \beta_{j}\right]\right)$ is hyperbolic: We may assume, by conjugating, that

$$
\rho_{1}^{j}\left(\left[\alpha_{j}, \beta_{j}\right]\right)=\rho_{2}^{j}\left(\left[\alpha_{j}, \beta_{j}\right]\right)=\left(\begin{array}{cc}
\lambda & 0 \\
0 & \lambda^{-1}
\end{array}\right)
$$


where $|\lambda|>1$. As $\rho_{1}^{j}$ and $\rho_{2}^{j}$ agree on $G_{j}$, we can assume that $\delta$ is not either $\alpha_{j}$ or $\beta_{j}$. Let

$$
\rho_{i}^{j}(\delta)=\left(\begin{array}{cc}
e_{i} & f_{i} \\
g_{i} & h_{i}
\end{array}\right)
$$

where $e_{i} h_{i}-f_{i} g_{i}=1$. Moreover, since the traces agree on generators,

$$
e_{1}+h_{1}=e_{2}+h_{2} \text {. }
$$

Suppose that $\gamma \in G_{j}$ and $\gamma, \gamma \delta \in W\left(\alpha_{1}\right)$. (We can choose either $\gamma=\beta_{j}$ or $\gamma=\beta_{j}^{-1}$ depending on the orientations on the curves, see figure 2$)$. Let

$$
\rho_{1}^{j}(\gamma)=\rho_{2}^{j}(\gamma)=\left(\begin{array}{ll}
a & b \\
c & d
\end{array}\right)
$$

where $a d-b c=1$.

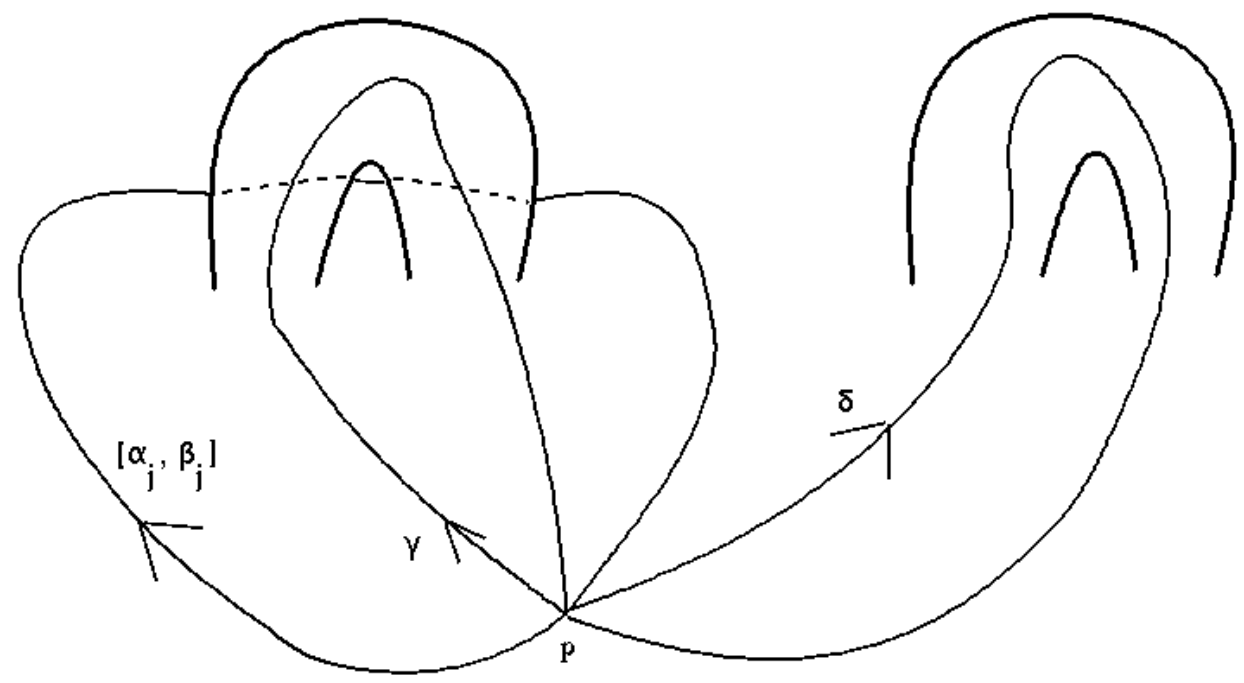

Figure 2. Curves $\gamma, \delta$ and $\left[\alpha_{j}, \beta_{j}\right]$

Let $\gamma_{n}=\left[\alpha_{j}, \beta_{j}\right]^{n} \gamma\left[\alpha_{j}, \beta_{j}\right]^{-n} \delta$, which also lies in $W\left(\alpha_{1}\right)$, since it is the image of $\gamma \delta$ under the $n$-fold Dehn twist about $\left[\alpha_{j}, \beta_{j}\right]$. So, by assumption,

$$
\operatorname{Tr}\left(\rho_{1}^{j}\left(\gamma_{n}\right)\right)= \pm \operatorname{Tr}\left(\rho_{2}^{j}\left(\gamma_{n}\right)\right) \text {. }
$$

By expanding, we see that, for all $n$,

$$
a e_{1}+d h_{1}+b g_{1} \lambda^{2 n}+c f_{1} \lambda^{-2 n}= \pm\left(a e_{2}+d h_{2}+b g_{2} \lambda^{2 n}+c f_{2} \lambda^{-2 n}\right) .
$$

Thus there exists an increasing subsequence where the traces either all agree or all differ up to sign. 
If there exists an increasing sequence $\left\{n_{j}\right\}$ such that the traces all agree, then

$$
a e_{1}+d h_{1}+b g_{1} \lambda^{2 n_{j}}+c f_{1} \lambda^{-2 n_{j}}=a e_{2}+d h_{2}+b g_{2} \lambda^{2 n_{j}}+c f_{2} \lambda^{-2 n_{j}}
$$

for all $n_{j}$. Dividing the above equation by $\lambda^{2 n_{j}}$ and taking a limit as $j \rightarrow \infty$, we see that $b g_{1}=b g_{2}$. Since $b \neq 0$, by Lemma $2.2, g_{1}=g_{2}$. Thus,

$$
a e_{1}+d h_{1}+c f_{1} \lambda^{-2 n_{j}}=a e_{2}+d h_{2}+c f_{2} \lambda^{-2 n_{j}}
$$

and we see, by taking the limit as $n_{j} \rightarrow \infty$, that $a e_{1}+d h_{1}=a e_{2}+d h_{2}$. Finally, since $c f_{1} \lambda^{-2 n j}=c f_{2} \lambda^{-2 n j}$ and $\lambda$ and $c$ are non-zero, $f_{1}=f_{2}$. Summarizing, we have

$$
a e_{1}+d h_{1}=a e_{2}+d h_{2}, \quad f_{1}=f_{2}, \quad \text { and } g_{1}=g_{2} .
$$

Similarly, if there exists an increasing sequence $\left\{n_{j}\right\}$ such that the traces all disagree then,

$$
a e_{1}+d h_{1}+b g_{1} \lambda^{2 n_{j}}+c f_{1} \lambda^{-2 n_{j}}=-\left(a e_{2}+d h_{2}+b g_{2} \lambda^{2 n_{j}}+c f_{2} \lambda^{-2 n_{j}}\right)
$$

for all $n_{j}$. Taking limits as above, we conclude that

$$
a e_{1}+d h_{1}=-\left(a e_{2}+d h_{2}\right), \quad f_{1}=-f_{2}, \quad \text { and } \quad g_{1}=-g_{2} .
$$

Thus given any $\gamma \in G_{j}$ such that $\gamma, \gamma \delta \in W\left(\alpha_{1}\right)$, then $\gamma$ either satisfies equation (1) or (2). Since $f_{i}$ and $g_{i}$ are non-zero, we conclude that, with the above normalization, either

(a) equation (1) holds for all $\gamma \in G_{j}$ such that $\gamma, \gamma \delta \in W\left(\alpha_{1}\right)$, or

(b) equation (2) holds for all $\gamma \in G_{j}$ such that $\gamma, \gamma \delta \in W\left(\alpha_{1}\right)$.

Case Ia): Equation (1) holds for all $\gamma \in G_{j}$ such that $\gamma, \gamma \delta \in W\left(\alpha_{1}\right)$. Choose $\gamma \in G_{j}$ such that $\gamma, \gamma \delta \in W\left(\alpha_{1}\right)$ and let

$$
\rho_{1}^{j}(\gamma)=\rho_{2}^{j}(\gamma)=\left(\begin{array}{ll}
a & b \\
c & d
\end{array}\right)
$$

where $a d-b c=1$. Then, by equation (1),

$$
a e_{1}+d h_{1}=a e_{2}+d h_{2}, \quad f_{1}=f_{2}, \quad \text { and } \quad g_{1}=g_{2} .
$$

Since $e_{i} h_{i}-f_{i} g_{i}=1$, we conclude that $e_{1} h_{1}=e_{2} h_{2}$, so, since we also have $e_{1}+h_{1}=e_{2}+h_{2}$,

$$
\left(x-e_{1}\right)\left(x-h_{1}\right)=x^{2}-2\left(e_{1}+h_{1}\right) x+e_{1} h_{1}=\left(x-e_{2}\right)\left(x-h_{2}\right),
$$

which implies that either (i) $e_{1}=e_{2}$ and $h_{1}=h_{2}$ or (ii) $e_{1}=h_{2}$ and $h_{1}=e_{2}$.

If $e_{1}=e_{2}$ and $h_{1}=h_{2}$, then, since we already know that $f_{1}=f_{2}$ and $g_{1}=g_{2}$, we may conclude that $\rho_{1}^{j}(\delta)=\rho_{2}^{j}(\delta)$ and we are in case (1).

If $e_{1}=h_{2}$ and $h_{1}=e_{2}$, then, since $a e_{1}+d h_{1}=a e_{2}+d h_{2}$, we conclude that

$$
(a-d)\left(e_{1}-h_{1}\right)=0 .
$$

If $a \neq d$, then this implies that $e_{1}=h_{1}$, so $e_{1}=e_{2}$. Since we already know that $f_{1}=f_{2}$ and $g_{1}=g_{2}$, and all the matrix co-efficients are non-zero, we may conclude that $\rho_{1}^{j}(\delta)=\rho_{2}^{j}(\delta)$, so we are again in case (1). 
In order to conclude that we are in case (1), it only remains to check that we can choose $\gamma_{0} \in G_{j}$, so that $\gamma_{0}, \gamma_{0} \delta \in W\left(\alpha_{1}\right)$ and

$$
\rho_{1}^{j}\left(\gamma_{0}\right)=\rho_{2}^{j}\left(\gamma_{0}\right)=\left(\begin{array}{cc}
a_{0} & b_{0} \\
c_{0} & d_{0}
\end{array}\right) \text {. }
$$

where $a_{0} \neq d_{0}$.

We may assume that we have initially chosen $\gamma$ to be either $\beta_{j}^{-1}$ or $\beta_{j}$, and that, with the above notation, $a=d$. We may then choose $\gamma_{0}$ to be the element in $\left\{\left[\alpha_{j}, \beta_{j}\right] \gamma,\left[\alpha_{j}, \beta_{j}\right]^{-1} \gamma\right\}$ which is simple and has the property that $\gamma_{0}, \gamma_{0} \delta \in W\left(\alpha_{1}\right)$ (see Figure 3). Observe that either $a_{0}=\lambda a$ and $d_{0}=\lambda^{-1} d$ or $a_{0}=\lambda^{-1} a$ and $d_{0}=\lambda d$, so $a_{0} \neq d_{0}$. This choice of $\gamma_{0}$ allows us to complete the proof.

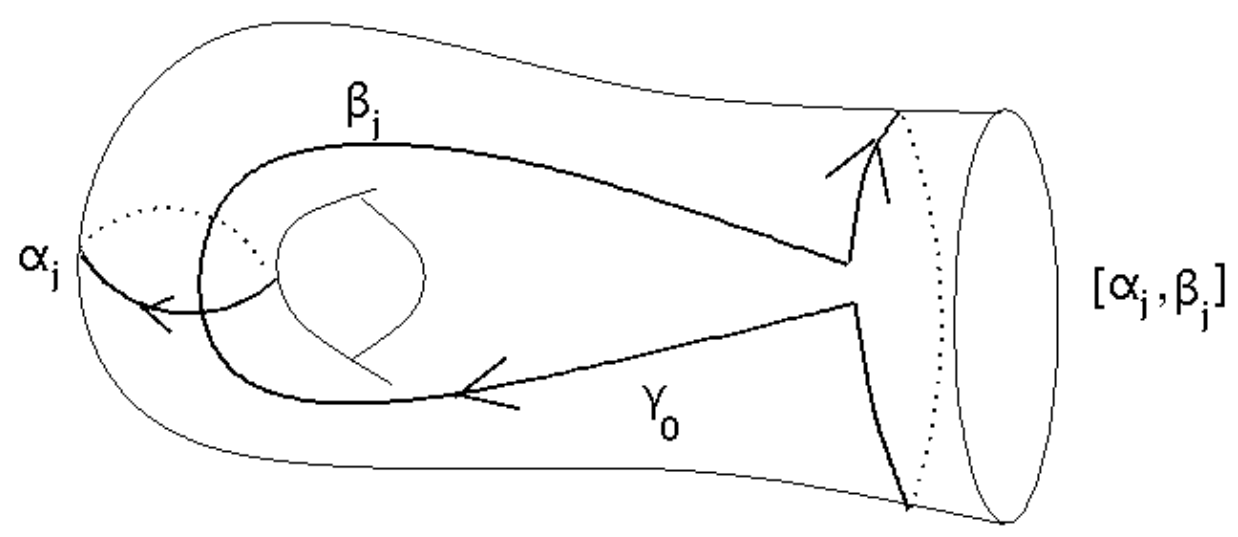

Figure 3. The curve $\gamma_{0}$

Case Ib): Equation (2) holds for all $\gamma \in G_{j}$ such that $\gamma, \gamma \delta \in W\left(\alpha_{1}\right)$. Choose $\gamma \in G_{j}$ so that $\gamma, \gamma \delta \in W\left(\alpha_{1}\right)$ and let

$$
\rho_{1}^{j}(\gamma)=\rho_{2}^{j}(\gamma)=\left(\begin{array}{ll}
a & b \\
c & d
\end{array}\right)
$$

where $a d-b c=1$. Then, by equation (2).

$$
a e_{1}+d h_{1}=-\left(a e_{2}+d h_{2}\right), \quad f_{1}=-f_{2}, \quad \text { and } \quad g_{1}=-g_{2} .
$$

As $e_{i} h_{i}-f_{i} g_{i}=1$, we conclude that $e_{1} h_{1}=e_{2} h_{2}$. Since $e_{1} h_{1}=e_{2} h_{2}$ and $e_{1}+h_{1}=e_{2}+h_{2}$, we may conclude, just as in Case Ia, that either (i) $e_{1}=h_{2}$ and $h_{1}=e_{2}$ or (ii) $e_{1}=e_{2}$, and $h_{1}=h_{2}$.

If $e_{1}=h_{2}$ and $h_{1}=e_{2}$, then, since $a e_{1}+d h_{1}=-\left(a e_{2}+d h_{2}\right)$, we see that

$$
\operatorname{Tr}\left(\rho_{1}^{j}(\gamma)\right) \operatorname{Tr}\left(\rho_{1}^{j}(\delta)\right)=(a+d)\left(e_{1}+h_{1}\right)=a e_{1}+d h_{1}+a e_{2}+d h_{2}=0
$$


which is impossible since both traces are non-zero. Therefore, $e_{1}=e_{2}$ and $h_{1}=h_{2}$, so. since $f_{1}=-f_{2}$ and $g_{1}=-g_{2}$,

$$
\rho_{2}^{j}(\delta)=\left(\begin{array}{cc}
e_{1} & -f_{1} \\
-g_{1} & h_{1}
\end{array}\right)=\left(\begin{array}{cc}
i & 0 \\
0 & -i
\end{array}\right)\left(\begin{array}{cc}
e_{1} & f_{1} \\
g_{1} & h_{1}
\end{array}\right)\left(\begin{array}{cc}
-i & 0 \\
0 & i
\end{array}\right)=R_{j} \rho_{1}^{j}(\delta) R_{j}^{-1}
$$

so $\rho_{1}^{j}(\delta)=R_{j} \rho_{2}^{j}(\delta) R_{j}^{-1}$ and $R_{j}$ is a lift of the rotation of order two about the axis of $\rho_{1}^{j}\left(\left[\alpha_{j}, \beta_{j}\right]\right)$.

Moreover, since $a e_{1}+d h_{1}=-\left(a e_{2}+d h_{2}\right)=-\left(a e_{1}+d h_{1}\right)$, we see that $a e_{1}+d h_{1}=0$, so

$$
\frac{e_{1}}{h_{1}}=-\frac{d}{a}
$$

and we are in case (2).

Case II: $\rho_{1}^{j}\left(\left[\alpha_{j}, \beta_{j}\right]\right)$ is parabolic: Choose $\gamma \in G_{j}$ so that $\gamma, \gamma \delta \in W\left(\alpha_{1}\right)$. We may conjugate so that

$$
\rho_{1}^{j}\left(\left[\alpha_{j}, \beta_{j}\right]\right)=\rho_{2}^{j}\left(\left[\alpha_{j}, \beta_{j}\right]\right)=\left(\begin{array}{cc}
1 & 1 \\
0 & 1
\end{array}\right)
$$

and 0 is a fixed points of $\rho_{1}^{j}(\gamma)=\rho_{2}^{j}(\gamma)$, so

$$
\rho_{1}^{j}(\gamma)=\rho_{2}^{j}(\gamma)=\left(\begin{array}{ll}
a & 0 \\
c & d
\end{array}\right)
$$

where $a d=1$. So, $a$ and $d$ are non-zero and $c$ is non-zero, since otherwise $\rho_{1}^{i}\left(\left[\alpha_{i}, \beta_{i}\right]\right)$ and $\rho_{1}^{i}(\gamma)$ have a common fixed point.

Let

$$
\rho_{i}^{j}(\delta)=\left(\begin{array}{cc}
e_{i} & f_{i} \\
g_{i} & h_{i}
\end{array}\right)
$$

where $e_{i} h_{i}-f_{i} g_{i}=1$ and all the matrix coefficients are non-zero. Moreover, since the lifts are trace normalized with respect to $\mathcal{S}$,

$$
e_{1}+h_{1}=e_{2}+h_{2} \text {. }
$$

Again, let $\gamma_{n}=\left[\alpha_{i}, \beta_{i}\right]^{n} \gamma\left[\alpha_{i}, \beta_{i}\right]^{-n} \delta$, which lies in $W\left(\alpha_{1}\right)$, so

$$
\operatorname{Tr}\left(\rho_{1}^{i}\left(\gamma_{n}\right)\right)= \pm \operatorname{Tr}\left(\rho_{2}^{i}\left(\gamma_{n}\right)\right) \text {. }
$$

Expanding, we obtain

$(a+n c) e_{1}+\left(n(d-a)-n^{2} c\right) g_{1}+c f_{i}+(d-n c) h_{1}= \pm\left((a+n c) e_{2}+\left(n(d-a)-n^{2} c\right) g_{2}+c f_{2}+(d-n c) h_{2}\right)$.

If there are infinitely many $n$ where the traces differ, then, by dividing by $n^{2}$ and taking limits, we conclude that $c g_{1}=-c g_{2}$, so, since $c \neq 0, g_{1}=-g_{2}$. By, successively taking limits, we further conclude that

$$
c\left(e_{1}-h_{1}\right)=-c\left(e_{2}-h_{2}\right) \quad \text { and } \quad a e_{1}+c f_{1}+d h_{1}=-a e_{2}-c f_{2}-d h_{2}
$$

Since $c \neq 0$, we see that $e_{1}-h_{1}=-e_{2}+h_{2}$. As $e_{1}+h_{1}=e_{2}+h_{2}$, this implies that $e_{1}=h_{2}$ and $h_{1}=e_{2}$. Therefore,

$$
f_{1} g_{1}=e_{1} h_{1}-1=e_{2} h_{2}-1=f_{2} g_{2}=-f_{2} g_{1} .
$$


As $\rho_{i}^{j}(\delta)$ does not have a fixed point at $\infty, g_{1}$ must be non-zero, so $f_{1}=-f_{2}$. Since $a e_{1}+c f_{1}+d h_{1}=-a e_{2}-c f_{2}-d h_{2}$, this implies that

$$
\operatorname{Tr}(\gamma) \operatorname{Tr}(\delta)=(a+d)\left(e_{1}+h_{1}\right)=0
$$

which is a contradiction, since both traces are non-zero.

Therefore, there are infinitely many $n$ where the traces agree, so, taking limits as above, we see that

$$
g_{1}=g_{2}, \quad c\left(e_{1}-h_{1}\right)=c\left(e_{2}-h_{2}\right), \quad \text { and } \quad a e_{1}+c f_{1}+d h_{1}=a e_{2}+c f_{2}+d h_{2} .
$$

Since $c \neq 0, e_{1}-h_{1}=e_{2}-h_{2}$, which we may combine with the fact that $e_{1}+h_{1}=e_{2}+h_{2}$, to conclude that $e_{1}=e_{2}$ and $h_{1}=h_{2}$. Therefore,

$$
f_{1} g_{1}=e_{1} h_{1}-1=e_{2} h_{2}-1=f_{2} g_{2}=f_{2} g_{1}
$$

which implies that $f_{1}=f_{2}$, so $\rho_{1}^{j}(\delta)=\rho_{2}^{j}(\delta)$, which implies that we are in case (1).

We now refine our analysis of trace normalized lifts which agree on $G_{j}$ to show that, for all $k$, they either agree on $G_{k}$ or differ by a rotation in the axis of the image of the commutator in $G_{j}$.

Lemma 3.4. Suppose that $\rho_{1}: \pi_{1}(S) \rightarrow \mathrm{SL}(2, \mathbb{C})$ and $\rho_{2}: \pi_{1}(S) \rightarrow \mathrm{SL}(2, \mathbb{C})$ are trace normalized lifts, with respect to a standard generating set $\mathcal{S}$, of Kleinian surface groups with the same marked complex length spectrum on $W\left(\alpha_{1}\right)$. If $j, k \in\{1, \ldots, g\}$ and $\rho_{1}^{j}$ and $\rho_{2}^{j}$ agree on $G_{j}=<\alpha_{j}, \beta_{j}>$, then either

(1) $\rho_{1}^{j}$ and $\rho_{2}^{j}$ agree on $G_{k}$, or

(2) $\rho_{1}^{j}\left(\left[\alpha_{j}, \beta_{j}\right]\right)$ is hyperbolic and $\left.\rho_{1}^{j}\right|_{G_{k}}=\left.\left(R_{j} \rho_{2}^{j} R_{j}^{-1}\right)\right|_{G_{k}}$ where $R_{j}$ is a lift of the rotation of order two about the axis of $\rho_{1}^{j}\left(\left[\alpha_{j}, \beta_{j}\right]\right)$.

Proof. If neither (1) or (2) holds, then Lemma 3.3 implies that, perhaps after switching $\alpha_{k}$ and $\beta_{k}, \rho_{1}^{j}\left(\left[\alpha_{j}, \beta_{j}\right]\right)$ is hyperbolic,

$$
\rho_{2}^{j}\left(\alpha_{k}\right)=\rho_{1}^{j}\left(\alpha_{2}\right) \quad \text { and } \quad \rho_{2}^{j}\left(\beta_{k}\right)=R_{j} \rho_{1}\left(\beta_{k}\right) R_{j}^{-1}
$$

where $R_{j}$ is a lift of the rotation of order two about the axis of $\rho_{1}^{j}\left(\left[\alpha_{j}, \beta_{j}\right]\right)$.

Lemma 3.2 implies that there exists $K \in \mathrm{SL}(2, \mathbb{C})$ so that $\rho_{1}^{j}$ and $K \rho_{2}^{j} K^{-1}$ agree on $G_{k}$. If $K= \pm I$, then $\rho_{1}^{j}$ and $\rho_{2}^{j}=K \rho_{2}^{j} K^{-1}$ agree on $G_{k}$ and we are in case (1). So, we may assume that $K \neq \pm I$ and

$\rho_{1}^{j}\left(\alpha_{k}\right)=K \rho_{2}^{j}\left(\alpha_{k}\right) K^{-1}=K \rho_{1}^{j}\left(\alpha_{k}\right) K^{-1} \quad$ and $\quad \rho_{1}^{j}\left(\beta_{k}\right)=K \rho_{2}^{j}\left(\beta_{k}\right) K^{-1}=K R_{j} \rho_{1}^{j}\left(\beta_{k}\right) R_{j}^{-1} K^{-1}$.

It follows that $K$ fixes the fixed points of $\rho_{1}^{j}\left(\alpha_{k}\right)$ and that $K R_{j}$ fixes the fixed points of $\rho_{1}^{j}\left(\beta_{k}\right)$.

If $\rho_{1}^{j}$ and $K \rho_{2}^{j} K^{-1}$ agree on either $\alpha_{j}$ or $\beta_{j}$, then, since $\rho_{1}^{j}$ and $\rho_{2}^{j}$ agree on $G_{j}$, either

$$
\rho_{1}^{j}\left(\alpha_{j}\right)=K \rho_{2}^{j}\left(\alpha_{j}\right) K^{-1}=K \rho_{1}^{j}\left(\alpha_{j}\right) K^{-1} \text { or } \rho_{1}^{j}\left(\beta_{j}\right)=K \rho_{2}^{j}\left(\beta_{j}\right) K^{-1}=K \rho_{1}^{j}\left(\beta_{j}\right) K^{-1}
$$


which implies that $K$ fixes the fixed points of either $\rho_{1}^{j}\left(\alpha_{j}\right)$ or $\rho_{1}^{j}\left(\beta_{j}\right)$. But, since $K$ fixes the fixed points of $\rho_{1}^{j}\left(\alpha_{k}\right)$, and the fixed points of $\rho_{1}^{j}\left(\alpha_{k}\right), \rho_{1}^{j}\left(\alpha_{j}\right)$ and $\rho_{1}^{j}\left(\beta_{j}\right)$ are all distinct, this implies that $K= \pm I$, which is a contradiction.

Therefore, $\rho_{1}^{j}$ and $K \rho_{2}^{j} K^{-1}$ disagree on both $\alpha_{j}$ and $\beta_{j}$. Lemma 3.3 then implies that $\rho_{1}^{j}\left(\left[\alpha_{k}, \beta_{k}\right]\right)$ is hyperbolic and

$$
\rho_{1}^{j}\left(\alpha_{j}\right)=R_{k} K \rho_{2}\left(\alpha_{j}\right) K^{-1} R_{k}^{-1} \quad \text { and } \quad \rho_{1}^{j}\left(\beta_{j}\right)=R_{k} K \rho_{2}^{j}\left(\beta_{j}\right) K^{-1} R_{k}^{-1}
$$

where $R_{k}$ is a lift of the rotation of order two about the axis of $\rho_{1}^{j}\left(\left[\alpha_{k}, \beta_{k}\right]\right)$. Therefore, $\rho_{1}^{j}$ and $R_{k} K \rho_{2}^{j} K^{-1} R_{k}^{-1}$ agree on $G_{j}$. Since $\rho_{1}^{j}$ and $\rho_{2}^{j}$ agree on $G_{j}$ this implies that $R_{k} K= \pm I$, so $K= \pm R_{k}^{-1}$. Therefore, $K$ fixes the fixed points of $\rho_{1}^{j}\left(\left[\alpha_{k}, \beta_{k}\right]\right)$. However, since we already know that $K$ fixes the fixed points of $\rho_{1}^{j}\left(\alpha_{k}\right)$, this implies that $K= \pm I$, which is again a contradiction.

It is now relatively simple to use Lemma 3.4 to complete the proof when $S$ has genus at least three.

Lemma 3.5. Suppose that $S$ has genus $g \geq 3$ and that $\tilde{\rho}_{1}: \pi_{1}(S) \rightarrow \operatorname{SL}(2, \mathbb{C})$ and $\tilde{\rho}_{2}: \pi_{1}(S) \rightarrow \mathrm{SL}(2, \mathbb{C})$ are trace normalized lifts, with respect to a standard generating set $\mathcal{S}$, of Kleinian surface groups with the same marked complex length spectrum on $W\left(\alpha_{1}\right)$. Then, $\tilde{\rho}_{1}$ is conjugate to $\tilde{\rho}_{2}$ in $\mathrm{SL}(2, \mathbb{C})$.

Proof. Lemma 3.2 implies that we may choose conjugates $\rho_{1}^{1}$ and $\rho_{2}^{1}$ of $\tilde{\rho}_{1}$ and $\tilde{\rho}_{2}$ which agree on $G_{1}=<\alpha_{j}, \beta_{j}>$. Lemma 3.4 implies that for all $j>1$, the restrictions of $\rho_{1}^{1}$ and $\rho_{2}^{1}$ to $G_{j}$ either agree or are conjugate by $R_{1}$, where $R_{1}$ is a lift of the rotation of order two about the axis of $\rho_{1}^{1}\left(\left[\alpha_{1}, \beta_{1}\right]\right)$. If $\rho_{1}^{1} \neq \rho_{2}^{1}$, we may assume without loss of generality that $\left.\rho_{1}^{1}\right|_{G_{2}}=\left.\left(R_{1} \rho_{2}^{1} R_{1}^{-1}\right)\right|_{G_{2}}$.

Lemma 3.4 implies that either (1) $\rho_{1}^{1}$ and $R_{1} \rho_{2}^{1} R_{1}^{-1}$ agree on $G_{1}$, or $(2) \rho_{2}^{1}\left(\left[\alpha_{2}, \beta_{2}\right]\right)$ is hyperbolic and $\rho_{1}^{1}$ and $R_{1} \rho_{2}^{1} R_{1}^{-1}$ are conjugate by $R_{2}$ on $G_{1}$ where $R_{2}$ is a lift of the rotation of order two about the axis of $\rho_{1}^{1}\left(\left[\alpha_{2}, \beta_{2}\right]\right)$.

If $\rho_{1}^{1}$ and $R_{1} \rho_{2}^{1} R_{1}^{-1}$ agree on $G_{1}$, then, since $\rho_{1}^{1}$ and $\rho_{2}^{1}$ agree on $G_{1}, R_{1}$ commutes with every element of $\rho_{1}^{1}\left(G_{1}\right)$. Since $\rho_{1}^{1}\left(G_{1}\right)$ is non-elementary, this implies that $R_{1}= \pm I$, which is a contradiction.

If $\rho_{1}^{1}$ and $R_{2}\left(R_{1} \rho_{2}^{1} R_{1}^{-1}\right) R_{2}^{-1}$ agree on $G_{1}$, then since $\rho_{1}^{1}$ and $\rho_{2}^{1}$ agree on $G_{1}$, we similarly conclude that $R_{2} R_{1}= \pm I$. So, $R_{2}= \pm R_{1}^{-1}$. This would imply that $\rho_{1}^{1}\left(\left[\alpha_{1}, \beta_{1}\right]\right)$ and $\rho_{1}^{1}\left(\left[\alpha_{2}, \beta_{2}\right]\right)$ have the same axis, so share fixed points, which is a contradiction unless $S$ has genus 2 and $\left[\alpha_{1}, \beta_{1}\right]=\left[\alpha_{2}, \beta_{2}\right]^{-1}$.

We now complete the proof by establishing our result in the genus two case.

Lemma 3.6. Suppose that $S$ has genus $g=2$ and that $\tilde{\rho}_{1}: \pi_{1}(S) \rightarrow \operatorname{SL}(2, \mathbb{C})$ and $\tilde{\rho}_{2}: \pi_{1}(S) \rightarrow \mathrm{SL}(2, \mathbb{C})$ are trace normalized lifts, with respect to a standard generating set $\mathcal{S}$, of Kleinian surface groups with the same marked complex length spectrum on $W\left(\alpha_{1}\right)$. Then, $\tilde{\rho}_{1}$ is conjugate to $\tilde{\rho}_{2}$ in $\mathrm{SL}(2, \mathbb{C})$. 
Proof. In this case $\pi_{1}(S)=<\alpha_{1}, \beta_{1}, \alpha_{2}, \beta_{2}>$ given by the standard oriented curves as in figure 4 . Lemma 3.2 implies that we may conjugate $\tilde{\rho}_{i}$ to $\rho_{i}^{1}$ so that $\rho_{1}^{1}$ and $\rho_{2}^{1}$ agree on $G_{1}$.

Suppose that $\rho_{1}^{1} \neq \rho_{2}^{1}$. Lemma 3.4 implies that $\rho_{1}\left(\left[\alpha_{1}, \beta_{1}\right]\right)$ is hyperbolic and $\rho_{1}^{1}$ and $R_{1} \rho_{2}^{1} R_{1}^{-1}$ agree on $G_{2}$ where $R_{1}$ is a lift of the rotation of order two about the axis of $\rho_{1}\left(\left[\alpha_{1}, \beta_{1}\right]\right)$. We may normalize so that

$$
\rho_{1}^{1}\left(\left[\alpha_{1}, \beta_{1}\right]\right)=\rho_{2}^{1}\left(\left[\alpha_{1}, \beta_{1}\right]\right)=\left(\begin{array}{cc}
\lambda & 0 \\
0 & \lambda^{-1}
\end{array}\right)
$$

where $|\lambda|>1$.

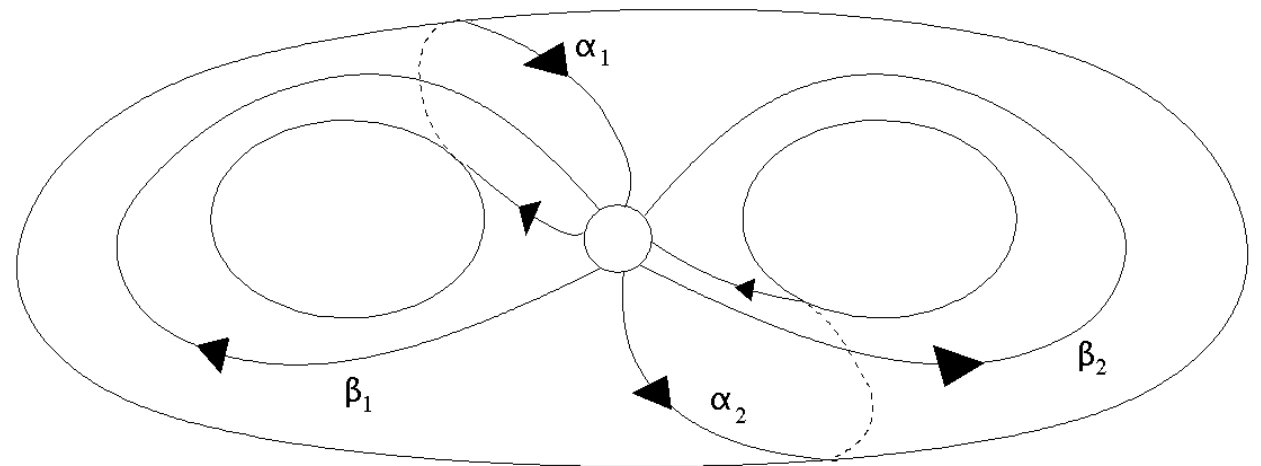

Figure 4. Surface of genus 2 with standard generators

Let $\gamma=\alpha_{1}^{-1}$. Notice that, assuming that elements of $\mathcal{S}$ are oriented as in figure $4, \gamma \alpha_{2}$ and $\gamma \beta_{2}$ are simple and lie in $W\left(\alpha_{1}\right)$. Define $A=\rho_{1}^{1}\left(\alpha_{1}^{-1}\right), C=\rho_{1}^{1}\left(\alpha_{2}\right)$, and $D=\rho_{1}^{1}\left(\beta_{2}\right)$, and adapt the convention that if a matrix is denoted by capital letter $X$, then it has coefficients $\left(x_{i j}\right)$. Since $\rho_{1}^{1}$ and $\rho_{2}^{1}$ differ on both $\alpha_{2}$ and $\beta_{2}$, Lemma 3.3 implies that

$$
\frac{c_{11}}{c_{22}}=\frac{d_{11}}{d_{22}}=-\frac{a_{22}}{a_{11}} .
$$

We now consider the element $\alpha_{2} \beta_{2} \in G_{2}$. We replace generators $\left\{\alpha_{2}, \beta_{2}\right\}$ by $\left\{\alpha_{2} \beta_{2}, \alpha_{2}^{-1}\right\}$ in $\mathcal{S}$ to form another standard generating set $\mathcal{S}^{\prime}=\left\{\alpha_{1}, \beta_{1}, \alpha_{2} \beta_{2}, \alpha_{2}^{-1}\right\}$ (we note that $\left.\left[\alpha_{2} \beta_{2}, \alpha_{2}^{-1}\right]=\left[\alpha_{2}, \beta_{2}\right]\right)$. The representations $\rho_{1}^{1}$ and $\rho_{2}^{1}$ agree on $G_{1}$ and their traces agree on $\mathcal{S}^{\prime}$. Notice that $\gamma\left(\alpha_{2} \beta_{2}\right) \in W\left(\alpha_{1}\right)$ (see figure 4) and let $E=C D=\rho_{1}\left(\alpha_{2} \beta_{2}\right)$. Since $\rho_{1}^{1}$ and $\rho_{2}^{1}$ agree on $G_{1}$ and disagree on $\alpha_{2} \beta_{2}$, Lemma 3.3 implies that

$$
\frac{e_{11}}{e_{22}}=-\frac{a_{22}}{a_{11}} .
$$

If $M=C D C^{-1} D^{-1}=\rho_{1}^{1}\left(\left[\alpha_{2}, \beta_{2}\right]^{-1}\right)=\rho_{1}^{1}\left(\left[\alpha_{1}, \beta_{1}\right]\right)$, then

$$
M=\left(\begin{array}{cc}
\lambda & 0 \\
0 & \lambda^{-1}
\end{array}\right)
$$


Since $D C=M^{-1} C D=M^{-1} E$ and $C^{-1}(C D) C=D C$,

$$
\operatorname{Tr}(D C)=\operatorname{Tr}(C D)=\operatorname{Tr}(E)=\operatorname{Tr}\left(M^{-1} E\right),
$$

so $e_{11}+e_{22}=\lambda^{-1} e_{11}+\lambda e_{22}$. Therefore

$$
\frac{c_{11}}{c_{22}}=\frac{d_{11}}{d_{22}}=\frac{e_{11}}{e_{22}}=\frac{(\lambda-1)}{\left(1-\lambda^{-1}\right)}=\lambda .
$$

Since $C D=M D C$,

$$
(C D)_{12}=c_{11} d_{12}+c_{12} d_{22}=(M D C)_{12}=\lambda(D C)_{12}=\lambda\left(d_{11} c_{12}+d_{12} c_{22}\right),
$$

so, since $c_{11}=\lambda c_{22}$ and $d_{11}=\lambda d_{22}$,

$$
\lambda c_{22} d_{12}+c_{12} d_{22}=\lambda\left(\lambda d_{22} c_{12}+d_{12} c_{22}\right),
$$

which implies that

$$
\left(\lambda^{2}-1\right) c_{12} d_{22}=0 .
$$

Thus, since $d_{22}$, and $c_{12}$ are both non-zero, $\lambda^{2}=1$ which is a contradiction. Therefore, it must be the case that $\rho_{1}^{1}=\rho_{2}^{1}$.

Remark: Observe that in the proof we only assume that there exists a simple nonseparating curve $\alpha$ such that $\rho_{1}$ and $\rho_{2}$ have the same or conjugate marked complex length spectrum on $W(\alpha)$. It then follows from the remark at the end of Section 2 that $\rho_{1}$ and $\rho_{2}$ are conjugate in the isometry group of $\mathbb{H}^{3}$ if they have the same marked length spectrum on all simple, non-separating curves.

\section{Reduction to Finitely many Simple CuRves}

In this brief section, we use our main result and standard algebraic techniques to show that there are finitely many simple curves whose lengths determine a Kleinian surface group up to conjugacy in the isometry group of $\mathbb{H}^{3}$. These same techniques were used by Kim [17] to observe that his marked length rigidity theorem (for all elements) implies that there are finitely many elements whose translation lengths determine an irreducible, non-elementary, nonparabolic representation into the isometry group of a rank one symmetric space up to conjugacy.

Corollary 1.2. If $S$ is a closed, connected, orientable surface of genus at least two, then there exists finitely many simple curves $\left\{\alpha_{1}, \ldots, \alpha_{n}\right\}$ on $S$ such that if $\rho_{1}: \pi_{1}(S) \rightarrow \operatorname{PSL}(2, \mathbb{C})$ and $\rho_{2}: \pi_{1}(S) \rightarrow \operatorname{PSL}(2, \mathbb{C})$ are Kleinian surface groups and $\ell_{\rho_{1}}\left(\alpha_{j}\right)=\ell_{\rho_{2}}\left(\alpha_{j}\right)$ for all $j=1, \ldots, n$, then $\rho_{1}$ is conjugate to either $\rho_{2}$ or $\bar{\rho}_{2}$.

Proof of Corollary 1.2: Recall that

$$
X(S)=\operatorname{Hom}\left(\pi_{1}(S), \operatorname{PSL}(2, \mathbb{C})\right) \times \operatorname{Hom}\left(\pi_{1}(S), \operatorname{PSL}(2, \mathbb{C})\right)
$$

is an affine algebraic variety, since it is a product of affine algebraic varieties. Let $\left\{\alpha_{j}\right\}_{j=1}^{\infty}$ be an enumeration of the simple curves on $S$. For each $k \in \mathbb{N}$, let

$$
Y_{k}=\left\{\left(\rho_{1}, \rho_{2}\right) \in X(S) \mid \ell_{\rho_{1}}\left(\alpha_{j}\right)=\ell_{\rho_{2}}\left(\alpha_{j}\right) \text { if } j=1, \ldots, k\right\} .
$$


Kim $\left[17\right.$, Lem. 2] shows that each $Y_{k}$ is an affine real algebraic sub-variety of $X(S)$. Let

$$
Y_{\infty}=\bigcap_{k=1}^{\infty} Y_{k} .
$$

The Noetherian property of polynomial rings implies that there exists $n \in \mathbb{N}$, such that $Y_{n}=Y_{\infty}$.

Our main result, Theorem 1.1, is equivalent to the statement that if $\rho_{1}$ and $\rho_{2}$ are Kleinian surface groups such that $\left(\rho_{1}, \rho_{2}\right) \in Y_{\infty}$, then $\rho_{1}$ and $\rho_{2}$ are conjugate in the isometry group of $\mathbb{H}^{3}$. It follows, that if $\rho_{1}$ and $\rho_{2}$ are Kleinian surface groups such that $\left(\rho_{1}, \rho_{2}\right) \in Y_{n}$, then $\rho_{1}$ and $\rho_{2}$ are conjugate in the isometry group of $\mathbb{H}^{3}$. However, this is simply a restatement of Corollary 1.2 , so we are done.

\section{ACYLINDRICAL 3-MANIFOLDS}

We now use our main result to prove our length rigidity theorem for acylindrical 3manifolds.

Theorem 1.3. If $M$ is a compact, acylindrical, hyperbolizable 3-manifold, and $\rho_{1}: \pi_{1}(M) \rightarrow \operatorname{PSL}(2, \mathbb{C})$ and $\rho_{2}: \pi_{1}(M) \rightarrow \operatorname{PSL}(2, \mathbb{C})$ are two discrete faithful representations, such that $\ell_{\rho_{1}}(\alpha)=$ $\ell_{\rho_{2}}(\alpha)$ if $\alpha \in \pi_{1}(M)$ is represented by a simple closed curve on $\partial M$, then $\rho_{1}$ is conjugate to either $\rho_{2}$ or $\bar{\rho}_{2}$.

In order to motivate the more general proof, we offer a sketch of the proof in the case that $M$ has connected boundary and $\rho_{1}$ and $\rho_{2}$ are both convex cocompact, i.e. each $N_{i}=$ $\mathbb{H}^{3} / \rho_{i}\left(\pi_{1}(M)\right)$ admits a conformal compactification with boundary $\partial_{c} N_{i}$. After perhaps replacing $\rho_{2}$ with a complex conjugate representation, we may assume by Johannson's Classification Theorem [15], that there exists an orientation-preserving homeomorphism $j: N_{1} \rightarrow N_{2}$ in the homotopy class determined by $\rho_{2} \circ \rho_{1}^{-1}$. In this case, work of Bers [2] implies that $\rho_{1}$ is conjugate to $\rho_{2}$ if and only if $j$ extends, up to isotopy, to a conformal homeomorphism $\hat{j}: \partial_{c} N_{1} \rightarrow \partial_{c} N_{2}$.

If the length spectra of $\rho_{1}$ and $\rho_{2}$ agree on simple curves on the boundary, our main result implies that the restrictions of $\rho_{1}$ and $\rho_{2}$ to the fundamental group of the boundary agree up to conjugacy in in the isometry group of $\mathbb{H}^{3}$. It follows that the conformal compactifications $N_{1}^{S}$ and $N_{2}^{S}$ of the covers of $N_{1}$ and $N_{2}$ associated to $\pi_{1}(S)$ are either conformal or anticonformally homeomorphic (by a homeomorphism in the homotopy class consistent with the identifications of their fundamental groups with $\pi_{1}(S)$.) Notice that $\partial_{c} N_{i}$ is identified with one component of the conformal boundary of $N_{i}^{S}$. If the conformal compactifications of $N_{1}^{S}$ and $N_{2}^{S}$ are conformally homeomorphic, then $j$ extends to a conformal homeomorphism between $\partial_{c} N_{1}$ and $\partial_{c} N_{2}$, so Bers' result implies that $\rho_{1}$ and $\rho_{2}$ are conjugate in $\operatorname{PSL}(2, \mathbb{C})$. If not, then one may use the Klein-Maskit combination Theorems (see Maskit [22]) to combine $\rho_{1}$ and (a conjugate of) $\rho_{2}$ to produce the holonomy representation of a hyperbolic structure on the double of $M$. Mostow's Rigidity Theorem [27] then implies that the involution of the double is an isometry, so the restriction of $\rho_{1}$ and $\rho_{2}$ to the boundary is Fuchsian, and the conformal structures on the boundary must agree. 
In the general case, we must organize the components of the boundary into those where the conformal structures agree and those where they disagree and replace the use of Bers' theorem and Mostow's Rigidity Theorem with applications of the Ending Lamination Theorem $[26,8]$.

Proof of Theorem 1.3: Suppose that $\rho_{1}: \pi_{1}(M) \rightarrow \operatorname{PSL}(2, \mathbb{C})$ and $\rho_{2}: \pi_{1}(M) \rightarrow \operatorname{PSL}(2, \mathbb{C})$ are two discrete faithful representations, such that $\ell_{\rho_{1}}(\alpha)=\ell_{\rho_{2}}(\alpha)$ if $\alpha \in \pi_{1}(M)$ is represented by a simple closed curve on $\partial M$. Let $N_{i}=\mathbb{H}^{3} / \rho_{i}\left(\pi_{1}(M)\right)$.

There exists a homotopy equivalence $h_{i}: M \rightarrow N_{i}$ in the homotopy class determined by $\rho_{i}$ (where we regard $\rho_{i}$ as an isomorphism between $\pi_{1}(M)$ and $\pi_{1}\left(N_{i}\right)=\rho_{i}\left(\pi_{1}(M)\right)$ ). Bonahon's Tameness Theorem [3] implies that $N_{i}$ may be identified with the interior of a compact 3-manifold $M_{i}$. Since $M$ is acylindrical, Johannson's Classification Theorem [15] implies that $h_{i}$ is homotopic to a homeomorphism. Therefore, there exists a homeomorphism $g: M_{1} \rightarrow M_{2}$ so that $g \circ h_{1}$ is homotopic to $h_{2}$, i.e. $g$ is in the homotopy class determined by $\rho_{2} \circ \rho_{1}^{-1}$. If $g$ is not orientation-preserving then we replace $\rho_{2}$ with $\bar{\rho}_{2}$. So, we may assume that $g$ is orientation-preserving.

Thurston associates to each $\rho_{i}$ a family of ending invariants, i.e. a multicurve $p_{i}$, called the parabolic locus, in the collection $\partial_{0} M_{i}$ of non-toroidal components of $\partial M_{i}$, and on each component of $\partial_{0} M_{i}-p_{i}$, either a hyperbolic structure of finite area (in which case the component is called geometrically finite) or a filling, geodesic lamination which admits a transverse measure of full support, called the ending lamination (in which case the component is called geometrically infinite), see $[26,8]$ for details. The Ending Lamination Theorem of Minsky [26] and Brock-Canary-Minsky [8] implies that $\rho_{1}$ is conjugate to $\rho_{2}$ in $\operatorname{PSL}(2, \mathbb{C})$ if and only if the ending invariants of $\rho_{1}$ agree with the ending invariants of $\rho_{2}$, i.e., up to isotopy of $g, g\left(p_{1}\right)=p_{2}$, the restriction of $g$ to each geometrically finite component of $\partial_{0} M_{1}-p_{1}$ is an isometry onto a geometrically finite component of $\partial_{0} M_{2}-p_{2}$, and if a geometrically infinite component of $\partial_{0} M_{1}-p_{1}$ has ending lamination $\lambda$, then $g(\lambda)$ is the ending lamination of a geometrically infinite component of $\partial_{0} M_{2}-p_{2}$.

Let $\left\{S_{1}, \ldots, S_{r}\right\}$ be the components of $\partial_{0} M_{1}$. Since $\ell_{\rho_{1}}(\alpha)=\ell_{\rho_{2}}(\alpha)$ if $\alpha \in \pi_{1}(M)$ is represented by a simple closed curve on $\partial M$, our main result, Theorem 1.1, implies that, for each $j,\left.\rho_{1}\right|_{\pi_{1}\left(S_{j}\right)}$ is conjugate to either $\rho_{2}$ or $\bar{\rho}_{2}$. If each $\left.\rho_{1}\right|_{\pi_{1}\left(S_{j}\right)}$ is conjugate to $\left.\rho_{2}\right|_{\pi_{1}\left(S_{j}\right)}$, then the ending invariants of $\rho_{1}$ agree with the ending invariants of $\rho_{2}$, so $\rho_{1}$ is conjugate to $\rho_{2}$ in $\operatorname{PSL}(2, \mathbb{C})$ and we are done.

If there exists $S_{j}$ so that $\left.\rho_{1}\right|_{\pi_{1}\left(S_{j}\right)}$ is conjugate to $\left.\bar{\rho}_{2}\right|_{\pi_{i}\left(S_{j}\right)}$, then we may re-order the components of $\partial M$, so that $\left.\rho_{1}\right|_{\pi_{1}\left(S_{j}\right)}$ is conjugate to $\left.\rho_{2}\right|_{\pi_{i}\left(S_{j}\right)}$ if and only if $j>q$ for some $q \in\{1, \ldots, r-1\}$. Let $N_{i}^{j}$ be the cover of $N_{i}$ associated to $\pi_{1}\left(S_{j}\right)$. Then, $N_{i}^{j}$ may be identified with the interior of $S_{j} \times[0,1]$ so that the end invariants of $N_{i}^{j}$ on $S_{j} \times\{1\}$ agree with the restriction of the end invariants of $N_{i}$ restricted to $S_{j}$. The Covering Theorem (see [10]) may be used to show that the restriction of the ending invariant of $N_{i}^{j}$ to $S_{j} \times\{0\}$ is a conformal structure on all of $S_{j} \times\{0\}$ (see the discussion in Richard Kent's proof of Thurston's Bounded Image Theorem [16, Thm. 41]). If $j \leq q$, then the ending invariant of $N_{1}^{j}$ on $S_{j} \times\{1\}$ agree with the end invariants of $N_{2}^{j}$ on $S_{j} \times\{0\}$, while the ending invariant 
of $N_{1}^{j}$ on $S_{j} \times\{0\}$ agree with the end invariants of $N_{2}^{j}$ on $S_{j} \times\{1\}$. It follows that, if $j \leq$, then the ending invariants of $N_{i}^{j}$ are a pair of hyperbolic structures on $S_{j} \times\{0\}$ and $S_{j} \times\{1\}$, so $\left.\rho_{i}\right|_{\pi_{1}\left(S_{j}\right)}$ is quasifuchsian, and the map from $S_{j} \times\{0,1\}$ to itself given by taking $(x, t)$ to $(x, 1-t)$ is isotopic to an isometry from the ending invariants of $N_{1}^{j}$ to the ending invariants of $N_{2}^{j}$.

Let $\hat{M}$ be the manifold obtained by doubling $M$ along $S_{1} \cup \cdots \cup S_{q-1}$. We first conjugate $\bar{\rho}_{2}$ so that $\rho_{1}\left(\pi_{1}\left(S_{1}\right)\right)=\bar{\rho}_{2}\left(\pi_{1}\left(S_{1}\right)\right)$. We may use the Klein-Maskit Combination Theorems [22] to see that the combination of $\rho_{1}\left(\pi_{1}(M)\right)$ and $\bar{\rho}_{2}\left(\pi_{1}(M)\right)$ gives rise to a hyperbolic structure on the double of $M$ along $S_{1}$. For each $j=2, \ldots, q$, let $A_{j} \in \operatorname{PSL}(2, \mathbb{C})$ be chosen so that $A_{j}$ conjugates $\left.\rho_{1}\right|_{\pi_{1}\left(S_{j}\right)}$ to $\left.\rho_{2}\right|_{\pi_{1}\left(S_{j}\right)}$. Then, the Klein-Maskit Combination Theorems give that the group generated by $\rho_{1}\left(\pi_{1}(M)\right), \rho_{2}\left(\pi_{1}(M)\right)$ and $\left\{A_{2}, \ldots A_{q}\right\}$ gives rise to a hyperbolic structure on $\hat{M}$ and so a representation $\hat{\rho}: \pi_{1}(\hat{M}) \rightarrow \operatorname{PSL}(2, \mathbb{C})$. (See Sections 8 and 9 of Morgan [25], in particular Theorem 9.4, for a discussion of the KleinMaskit Combination Theorems in a topological phrasing compatible with our application.) The obvious involution of $\hat{M}$ preserves the ending invariants of $\hat{N}=\mathbb{H}^{3} / \hat{\rho}\left(\pi_{1}(\hat{M})\right)$, so, by the Ending Lamination Theorem, there is an isometry of $\hat{N}$ realizing this involution. By restriction, $\rho_{1}$ is conjugate to $\rho_{2}$.

Remark: In the case that $\partial_{0} M$ is connected, Ian Agol astutely pointed out that one may rephrase this proof as an application of the fact that the square of the skinning map has a unique fixed point, see Morgan [25] and Kent [16] for a discussion of the skinning map.

\section{Renormalized Intersection Number}

We next show that the isometry group of the renormalized intersection number is generated by the (extended) mapping class group and complex conjugation. We begin by re-interpreting our renormalized intersection number in terms of the Patterson-Sullivan geodesic current, following Bridgeman [5] and Bridgeman-Taylor [7]. Since isometries of the renormalized intersection number are isometries of the pressure metric and the only degenerate vectors for the pressure metric are at points on the Fuchsian locus (see Bridgeman [5]), any isometry $f$ of the renormalized intersection number must preserve the Fuchsian locus. Since the restriction of the pressure metric to the Fuchsian locus is a multiple of the Weil-Petersson metric, we may apply Masur and Wolf's classification of isometries of the Weil-Petersson metric [23], to conclude that the restriction of $f$ to the Fuchsian locus agrees with the action of a mapping class $g$. We then use Bonahon's interpretation (see [4]) of the Thurston compactification of Teichmüller space in terms of geodesic currents, to show that if $\rho \in Q F(S)$, then $f(\rho)$ and $g(\rho)$ have proportional simple marked length spectrum. The proof is completed by showing that any two Kleinian surface groups with proportional simple marked length spectrum are conjugate in the isometry group of $\mathbb{H}^{3}$.

6.1. Geodesic Currents. Identify the space $G\left(\mathbb{H}^{2}\right)$ of geodesics in $\mathbb{H}^{2}$ with the open Möbius band $\left(\mathbb{S}_{\infty}^{1} \times \mathbb{S}_{\infty}^{1}-\right.$ diagonal $) / \mathbb{Z}_{2}$ by identifying a geodesic with its endpoints. A geodesic current on a hyperbolic surface $X=\mathbb{H}^{2} / \Gamma$ is a positive Borel measure on $G\left(\mathbb{H}^{2}\right)$ 
that is invariant under the action of $\Gamma$. For example, if $\alpha$ is a primitive closed geodesic on $X$, we may consider the geodesic current $C_{\alpha}$ given by taking the Dirac measure on the pre-image of $\alpha$ in $G\left(\mathbb{H}^{2}\right)$. Let $\mathcal{C}(X)$ be the space of geodesic currents defined for $X=\mathbb{H}^{2} / \Gamma$ with the weak*-topology. If $S$ is a closed surface of genus at least two, we identify $S$ with a fixed hyperbolic surface $X_{0}$ and let $\mathcal{C}(S)=\mathcal{C}\left(X_{0}\right)$.

Following Bonahon [4], Bridgeman and Taylor [7, Sec. 5] showed that there is a natural continuous, linear function

$$
L: \mathcal{C}(S) \times Q F(S) \rightarrow \mathbb{R},
$$

called the length function, with the property that if $r C_{\alpha}$ is a positive multiple of $C_{\alpha}$, then $L\left(r C_{\alpha}, \rho\right)=r \ell_{\rho}(\alpha)$. Since multiples of closed geodesics are dense in $\mathcal{C}(S)$ (see [3, Prop. $4.2]$ ), this property completely determines $L$.

Given $\rho \in Q F(S)$, Sullivan [31, Prop. 11, Thm. 21], following work of Patterson [28] in the Fuchsian case, used the Poincaré series to define a non-atomic $\Gamma$-invariant measure $\mu_{\rho}$ on $\partial_{\infty} \mathbb{H}^{3} \times \partial_{\infty} \mathbb{H}^{3}$, called the Patterson-Sullivan measure, which is supported on $\Lambda(\rho) \times \Lambda(\rho)$, where $\Lambda(\rho)$ is the limit set of $\rho\left(\pi_{1}(S)\right)$, such that $\Gamma$ acts ergodically on $\partial_{\infty} \mathbb{H}^{3} \times \partial_{\infty} \mathbb{H}^{3}$ with respect to $\mu_{\rho}$ (see also Sullivan [32, Thm. 3]). The representation $\rho$ induces a homeomorphism $f_{\rho}: S^{1} \rightarrow \Lambda(\rho)$, where we use our identification of $S$ with $X_{0}$ to identify $S^{1}$ with $\partial_{\infty} \mathbb{H}^{2}$. One then defines the Patterson-Sullivan current as the multiple $\psi(\rho) \in \mathcal{C}(S)$ of the pull-back $\left(f_{\rho} \times f_{\rho}\right)_{*}\left(\mu_{\rho}\right)$ so that $L(\psi(\rho), \rho)=1$. If $\rho$ is Fuchsian, then $\psi(\rho)$ is the Liouville geodesic current constructed by Bonahon [4] (see [14, Thm. 1]). Hamenstadt [14, Thm. 1] showed that the associated map

$$
\psi: Q F(S) \rightarrow \mathcal{C}(S)
$$

is continuous and injective. See Bridgeman [5], Bridgeman-Taylor [7] or Hamenstadt [14] for further discussion of the Patterson-Sullivan geodesic current.

Let $F(S)$ denote the space of Fuchsian representations in $Q F(S)$ and let $\mathcal{P C}(S)$ denote the space of projective classes of non-zero currents in $\mathcal{C}(S)$. Bonahon [4, Thm. 18] showed that if one considers the associated map into the space of projective geodesic currents $\bar{\psi}: F(S) \rightarrow \mathcal{P C}(S)$, then the closure of $\bar{\psi}(F(S))$ is $\bar{\psi}(F(S)) \cup P M L(S)$ where $P M L(S)$ is the space of projective classes of measured laminations. (We recall that the set $M L(S)$ of measured laminations in $\mathcal{C}(S)$ is exactly the closure of the set of positive multiples of currents associated to simple curves, see Bonahon [3, Sec. 4.3] for more details.)

We note that $L\left(\psi\left(\rho_{1}\right), \rho_{2}\right)$ can also be defined to be the length in $\rho_{2}$ of a random geodesic in $\rho_{1}$, i.e.

$$
L\left(\psi\left(\rho_{1}\right), \rho_{2}\right)=\lim _{T \rightarrow \infty} \frac{1}{\#\left\{[\gamma] \mid \ell_{\rho_{1}}(\gamma) \leq T\right\}} \sum_{\left\{[\gamma] \mid \ell_{\rho_{1}}(\gamma) \leq T\right\}} \frac{\left.\ell_{\rho_{2}}(\gamma)\right)}{\ell_{\rho_{1}}(\gamma)} .
$$

It follows that

$$
J\left(\rho_{1}, \rho_{2}\right)=\frac{h\left(\rho_{2}\right)}{h\left(\rho_{1}\right)} L\left(\psi\left(\rho_{1}\right), \rho_{2}\right) .
$$

In analogy with Thurston's formulation of the Weil-Petersson metric on Teichmüller space, and Bonahon's re-formulation in terms of geodesic currents [4, Thm. 19], Bridgeman 
and Taylor [7] consider, for each $\rho \in Q F(S)$, the function $J_{\rho}: Q F(S) \rightarrow \mathbb{R}$ given by $J_{\rho}(\sigma)=J(\rho, \sigma)$ and define a non-negative symmetric two-tensor $G$, called the pressure form, on the tangent bundle $T Q F(S)$, where $G_{\rho}$ on $T_{\rho} Q F(S)$ is the Hessian of $J_{\rho}$. By construction, the pressure form is invariant under the action of the (extended) mapping class group $\operatorname{Mod}^{*}(S)$ of $S$. It follows from the work of Wolpert [33] and Bonahon [4], that the restriction of the pressure form to the Fuchsian locus $F(S)$ is a multiple of the WeilPetersson metric. Motivated by the work of McMullen [24] in the setting of Teichmüller space, Bridgeman [5] showed that the pressure form is non-degenerate except along pure bending vectors based at points in the Fuchsian locus. Moreover, the pressure form gives rise to a path metric on $Q F(S)$, see [6, Cor. 1.7].

Remark: In the proof of Theorem 6.1 in [5], Bridgeman gives an expression for $L\left(\psi\left(\rho_{1}\right), \rho_{2}\right)$ in terms of equilibrium measures. Our equation (3) then follows from equations (12) and (13) in [6], see also the discussion in section 8 of [6]. If one prefers that the proof of Theorem 1.4 be self-contained, one can take $J$ to be defined by our equation (4) as is done in Bridgeman-Taylor [7] and Bridgeman [5].

6.2. Isometries of renormalized intersection number. We use the interpretation of renormalized intersection number in terms of geodesic currents to show that the restriction of an isometry $f$ of the renormalized intersection number to the Fuchsian locus $F(S)$ agrees with an element $g$ of the (extended) mapping class group. We further show that $f(\sigma)$ and $g(\sigma)$ have closely related length spectrum whenever $\sigma \in Q F(S)$.

Proposition 6.1. If $f: Q F(S) \rightarrow Q F(S)$ is a smooth isometry of $J$, then $f$ preserves the fuchsian locus $F(S)$ and there exists $g \in \operatorname{Mod}^{*}(S)$ such that $f$ and $g$ agree on the Fuchsian locus.

Moreover, if $\alpha$ is a simple curve on $S$ and $\sigma \in Q F(S)$, then

$$
h(g(\sigma)) \ell_{g(\sigma)}(\alpha)=h(f(\sigma)) \ell_{f(\sigma)}(\alpha) .
$$

Proof. Since $f$ is smooth and preserves $J$, one sees by differentiating that $f$ also preserves the pressure form $G$.

If $\sigma \in F(X)$ and $v$ is a pure-bending vector, then $G_{\sigma}(v, v)=0$, so $G_{f(\sigma)}\left(D f_{\sigma}(v), D f_{\sigma}(v)\right)=0$. Since, $f$ is an immersion, $D f_{\sigma}(v)=0$ which implies that $f(\sigma) \in F(S)$.

Since the restriction of $G$ to $F(S)$ is a multiple of the Weil-Petersson metric, the restriction of $f$ to the Fuchsian locus is an isometry of the Weil-Petersson metric. Masur and Wolf [23] proved every isometry of the Weil-Petersson metric is an element of $\operatorname{Mod}^{*}(S)$. So we may choose $g \in \operatorname{Mod}^{*}(S)$ so that $f$ and $g$ agree on the Fuchsian locus $F(S)$.

If $\rho \in F(S)$ and $\sigma \in Q F(S)$, then since

$$
J(\rho, \sigma)=J(f(\rho), f(\sigma))=J(g(\rho), g(\sigma)),
$$

and $h$ is constant on $F(S)$, we may conclude that

$$
\frac{L(\psi(g(\rho)), g(\sigma))}{L(\psi(f(\rho), f(\sigma))}=\frac{h(f(\sigma))}{h(g(\sigma))} .
$$


If $\alpha$ is a simple curve, let $\left\{\rho_{n}\right\}$ be a sequence in $F(S)$ so that $\left\{\bar{\psi}\left(f\left(\rho_{n}\right)\right)\right\}=\left\{\bar{\psi}\left(g\left(\rho_{n}\right)\right)\right\}$ converges to $\left[C_{\alpha}\right]$ in $\mathcal{P C}(S)$. Since $L$ is continuous,

$$
\frac{\ell_{g(\sigma)}(\alpha)}{\ell_{f(\sigma)}(\alpha)}=\lim \frac{L\left(\psi\left(g\left(\rho_{n}\right)\right), g(\sigma)\right)}{L\left(\psi\left(f\left(\rho_{n}\right)\right), f(\sigma)\right)}=\frac{h(f(\sigma))}{h(g(\sigma))}
$$

which establishes our claim.

6.3. Simply $k$-related Kleinian surface groups. We say that two Kleinian surface groups $\rho_{1}: \pi_{1}(S) \rightarrow \operatorname{PSL}(2, \mathbb{C})$ and $\rho_{2}: \pi_{1}(S) \rightarrow \operatorname{PSL}(2, \mathbb{C})$ are simply $k$-related, for some $k>0$, if

$$
\ell_{\rho_{1}}(\alpha)=k \ell_{\rho_{2}}(\alpha)
$$

whenever $\alpha$ is a simple closed curve on $S$.

Proposition 6.1 implies that if $f$ is an isometry of the renormalized intersection number, then there exists $g \in \operatorname{Mod}^{*}(S)$, such that $f(\sigma)$ and $g(\sigma)$ are simply $k$-related whenever $\sigma \in Q F(S)$. Theorem 1.4 will thus follow from the following strengthening of our simple length rigidity theorem.

Theorem 1.5: If $S$ is a closed, connected, orientable surface of genus at least two and $\rho_{1}: \pi_{1}(S) \rightarrow \operatorname{PSL}(2, \mathbb{C})$ and $\rho_{2}: \pi_{1}(S) \rightarrow \operatorname{PSL}(2, \mathbb{C})$ are simply $k$-related Kleinian surface groups, then $\rho_{1}$ is conjugate to either $\rho_{2}$ or $\bar{\rho}_{2}$.

Proof of Theorem 1.5: We first choose non-separating simple curves $\alpha$ and $\beta$ on $S$ which intersect exactly once, so that $\rho_{1}(\alpha), \rho_{1}(\beta), \rho_{2}(\alpha)$ and $\rho_{2}(\beta)$ are all hyperbolic. (One may do so, since, again by a result of Sullivan [30], only finitely many simple curves have parabolic images for $\rho_{1}$ or $\rho_{2}$.)

We can assume that $\rho_{1}$ and $\rho_{2}$ are $(\alpha, \beta)$-normalized, so

$$
\rho_{i}(\alpha)= \pm\left(\begin{array}{cc}
\lambda_{i} & 0 \\
0 & \lambda_{i}^{-1}
\end{array}\right)
$$

where $\left|\lambda_{i}\right|>1$, and

$$
\rho_{i}(\beta)= \pm\left(\begin{array}{cc}
a_{i} & b_{i} \\
c_{i} & d_{i}
\end{array}\right)
$$

where all the matrix coefficients are non-zero (see Lemma 2.2). Since $\rho_{1}$ and $\rho_{2}$ are simply $k$-related,

$$
\left|\lambda_{1}\right|=\left|\lambda_{2}\right|^{k}
$$

Notice that if $k=1$, then Theorem 1.5 follows immediately from our main result, Theorem 1.1. So we may assume, without loss of generality, that $k>1$.

Let $\left|\mu_{i}(n)\right|$ be the modulus of the eigenvalues of $\rho_{i}\left(\alpha^{n} \beta\right)$ with largest modulus. Since $\alpha^{n} \beta$ is simple for all $n,\left|\mu_{1}(n)\right|=\left|\mu_{2}(n)\right|^{k}$ for all $n$. Lemma 2.2 implies that

$$
\log \left|\mu_{i}(n)\right|=n \log \left|\lambda_{i}\right|+\log \left|a_{i}\right|+\Re\left(\lambda_{i}^{-2 n} \frac{b_{i} c_{i}}{a_{i}^{2}}\right)+O\left(\left|\lambda_{i}\right|^{-4 n}\right) .
$$


Since $\log \left|\mu_{1}(n)\right|=k \log \left|\mu_{2}(n)\right|$, after eliminating the leading terms (which are equal), we obtain

$$
\left.\log \left|a_{1}\right|+\Re\left(\lambda_{1}^{-2 n} \frac{b_{1} c_{1}}{a_{1}^{2}}\right)\right)+O\left(\left|\lambda_{1}\right|^{-4 n}\right)=k \log \left|a_{2}\right|+k \Re\left(\lambda_{2}^{-2 n} \frac{b_{2} c_{2}}{a_{2}^{2}}\right)+O\left(\left|\lambda_{2}\right|^{-4 n}\right) .
$$

Therefore, by considering the limits as $n \rightarrow \infty$, we see that

$$
\log \left|a_{1}\right|=k \log \left|a_{2}\right| \text {. }
$$

So, after subtracting the equal leading order terms,

$$
\Re\left(\lambda_{1}^{-2 n} \frac{b_{1} c_{1}}{a_{1}^{2}}\right)+O\left(\left|\lambda_{1}\right|^{-4 n}\right)=k \Re\left(\lambda_{2}^{-2 n} \frac{b_{2} c_{2}}{a_{2}^{2}}\right)+O\left(\left|\lambda_{2}\right|^{-4 n}\right) .
$$

for all $n$. If we multiplly both sides of the above equation by $\left|\lambda_{2}\right|^{2 n}$, then since $\frac{\left|\lambda_{1}\right|}{\left|\lambda_{2}\right|}>1$, the right hand side converges to 0 , and we see that

$$
\lim _{n \rightarrow \infty} \Re\left(\left(\frac{\lambda_{2}}{\left|\lambda_{2}\right|}\right)^{-2 n} \frac{b_{2} c_{2}}{a_{2}^{2}}\right)=0 .
$$

If we let

$$
u=\left(\frac{\lambda_{2}}{\left|\lambda_{2}\right|}\right)^{-2}=e^{i \theta} \quad \text { and } \quad v=\frac{b_{2} c_{2}}{a_{2}^{2}} \neq 0 .
$$

then we may rewrite (5) as

$$
\lim _{n \rightarrow \infty} \Re\left(u^{n} v\right)=0 .
$$

As we can always choose a sequence $\left\{n_{k}\right\}$ so that $\lim _{k \rightarrow \infty} u^{n_{k}}=1$, we see that

$$
\Re(v)=0 .
$$

If $\theta$ is an irrational multiple of $2 \pi$, then for all $z \in S^{1}$ there exists a sequence $\left\{n_{k}\right\}$ so that $\lim e^{n_{k} \theta}=z$, which implies that $\Re(z v)=0$. Therefore, in this case, as $v \neq 0$ then choosing $z=|v| / v$, we get $|v|=0$ a contradiction.

If $\theta$ is a rational multiple of $2 \pi$ and $u \neq \pm 1$, then there exists $\left\{n_{k}\right\}$ so that $u^{n_{k}}=u$ for all $k$, so $\Re(u v)=0$. Since $\Re(v)=0, v=i y$ from some $y \in \mathbb{R}$, so,

$$
\Re\left(e^{i \theta} v\right)=-\sin (\theta) y=0 .
$$

Thus, $y=0$, since $\sin (\theta) \neq 0$. It again follows that $v=0$ and we obtain a contradiction.

Therefore, we can assume that $u= \pm 1$, so $\lambda_{2}^{2}$ is real. It follows that if $\eta$ is a simple, non-separating curve on $S$, then $\lambda_{\rho_{2}}^{2}(\eta) \in \mathbb{R}$, so

$$
\operatorname{Tr}^{2}\left(\rho_{2}(\eta)\right)=\lambda_{\rho_{2}}^{2}(\eta)+2+\lambda_{\rho_{2}}^{-2}(\eta) \in \mathbb{R}
$$

In particular, since $\alpha^{n} \beta$ is simple and non-separating for all $n$, we see that

$$
\operatorname{Tr}^{2}\left(\rho_{2}\left(\alpha^{n} \beta\right)\right)=\lambda_{2}^{2 n} a_{2}^{2}+2 a_{2} d_{2}+\lambda_{2}^{-2 n} d_{2}^{2} \in \mathbb{R},
$$

so,

$$
\Im\left(\operatorname{Tr}^{2}\left(\rho_{2}\left(\alpha^{n} \beta\right)\right)\right)=\lambda_{2}^{2 n} \Im\left(a_{2}^{2}\right)+2 \Im\left(a_{2} d_{2}\right)+\lambda_{2}^{-2 n} \Im\left(d_{2}^{2}\right)=0
$$


for all $n$. Therefore,

$$
\lim _{n \rightarrow \infty} \frac{\Im\left(\operatorname{Tr}^{2}\left(\rho_{2}\left(\alpha^{n} \beta\right)\right)\right.}{\lambda_{2}^{2 n}}=\Im\left(a_{2}^{2}\right)=0,
$$

SO

$$
\lim _{n \rightarrow \infty} \Im\left(\operatorname{Tr}^{2}\left(\rho_{2}\left(\alpha^{n} \beta\right)\right)\right)=2 \Im\left(a_{2} d_{2}\right)=0 .
$$

Since $a_{2}^{2}$ and $a_{2} d_{2}$ are real, $v=\frac{b_{2} c_{2}}{a_{2}^{2}}=\frac{a_{2} d_{2}-1}{a_{2}^{2}}$ is real. Therefore, since we have already shown that $\Re(v)=0$, we see that $v=0$, which contradicts the fact that $a_{2}, b_{2}$ and $c_{2}$ are all non-zero. Since we have now achieved a contradiction in all cases where $k>1$, this completes the proof.

6.4. Proof of Theorem 1.4. If $f$ is an isometry of the renormalized intersection number, then Theorem 6.1 implies that there exists $g \in \operatorname{Mod}^{*}(S)$ such that $f$ and $g$ agree on $F(S)$ and $f(\sigma)$ and $g(\sigma)$ are simply $k$-related, where $k=\frac{h(f(\sigma))}{h(g(\sigma))}$, whenever $\sigma \in Q F(S)$. Theorem 1.5 implies that if $\sigma \in Q F(S)$, then either $f(\sigma)=g(\sigma)$ or $f(\sigma)=\tau(g(\sigma))$. Since $f$ is continuous, this implies that either $f=g$ or $f=\tau \circ g$. The result follows.

\section{REFERENCES}

[1] L. Bers, "Simultaneous uniformization," Bull. A.M.S. 66(1960), 94-97.

[2] L. Bers, "On moduli of Kleinian groups," Russian Math. Surveys 29 (1974), 88-102.

[3] F. Bonahon, "Bouts des variétés hyperboliques de dimension 3," Annals of Math. 124 (1986), 71-158.

[4] F. Bonahon "The geometry of Teichmüller space via geodesic currents," Invent. Math. 92(1988), 139162.

[5] M. Bridgeman, "Hausdorff dimension and the Weil-Petersson extension to quasifuchsian space," Geom. Top. 14(2010), 799-831.

[6] M. Bridgeman, R. Canary, F. Labourie, and A. Sambarino, "The pressure metric for Anosov representations," G.A.F.A., 25(2015), 1089-1179.

[7] M. Bridgeman and E. Taylor, "An extension of the Weil-Petersson metric to quasi-fuchsian space," Math Ann. 341(2008), 927-943.s

[8] J.F. Brock, R.D. Canary and Y.N. Minsky, "The Classification of Kleinian Surface Groups II: The Ending Lamination Conjecture," Annals of Math., 176 (2012), 1-149.

[9] M. Burger, "Intersection, the Manhattan curve and Patterson-Sullivan theory in rank 2," Internat. Math. Res. Notices 7(1993), 217-225.

[10] R.D. Canary, "A covering theorem for hyperbolic 3-manifolds and its applications," Topology 35(1996), 751-778.

[11] L. Charles and J. Marché, "Multicurves and regular functions on the representation variety of a surface in SU(2)," Comm. Math. Helv. 87(2012), 409-431.

[12] M. Culler, "Lifting representations to covering groups," Adv. Math. 59(1986), 64-70.

[13] M. Culler and P. Shalen, "Varieties of group representations and splittings of 3-manifolds," Annals of Math. 117(1983), 109-146.

[14] U. Hamenstädt, "Ergodic properties of function groups," Geom. Ded. 93(2002),163-176.

[15] K. Johannson, Homotopy Equivalences of 3-manifolds with Boundary, Lecture Notes in Mathematics, vol. 761, Springer-Verlag, 1979.

[16] R. Kent, "Skinning maps," Duke Math. J. 151(2010), 279-336.

[17] I. Kim, "Coordinates of the representation space in the semisimple Lie group of rank one," Bull. Austral. Math. Soc. 58(1998), 435-444. 
[18] I. Kim, "Marked length rigidity of rank one symmetric spaces and their product," Topology 40(2001), 1295-1323.

[19] C. Kourouniotis, "Complex length coordinates for quasi-fuchsian groups," Mathematika 41(1994), 173188

[20] I. Kra, "On lifting Kleinian groups to $\mathrm{SL}(2, \mathbb{C})$," in Differential geometry and complex analysis, Springer-Verlag, 1985, 181-193.

[21] J. Marché and M. Wolff, "The modular action on PSL(2, R)-characters in genus 2," Duke Math. J., to appear.

[22] B. Maskit, "On Klein's combination theorem, III," in Advances in the theory of Riemann surfaces, Ann. of Math. Studies 66(1971), 297-316.

[23] H. Masur and M Wolf, "The Weil-Petersson isometry group," Geom. Ded. 93(2002), 177-190

[24] C. McMullen, "Thermodynamics, dimension and the Weil-Petersson metric," Invent. Math. 173(2008), $365-425$.

[25] J. Morgan, "On Thurston's uniformization theorem for three dimensional manifolds," in The Smith Conjecture, Academic Press, 1984, 37-125.

[26] Y. Minsky, "The classification of Kleinian surface groups I: models and bounds," Annals of Math. 171 (2010), 1-107.

[27] G. D. Mostow, "Quasiconformal mappings in $n$-space and the rigidity of hyperbolic space forms," Publ. I.H.E.S. 34(1968), 53-104.

[28] S. Patterson, "The limit set of a fuchsian group," Acta Math. 136(1976), 241-273.

[29] H. Royden, "Automorphisms and isometries of Teichmüller spaces," in Advances in the theory of Riemann surfaces, Annals of Math. Studies, Vol. 66(1970), 369-383.

[30] D. Sullivan, "A finiteness theorem for cusps," Acta Math. 147(1981), 289-299.

[31] D. Sullivan, "The density at infinity of a discrete group of hyperbolic motions," Publ. Math. I.H.E.S. 50 (1979), 171-202.

[32] D. Sullivan, "Entropy, Hausdorff measures old and new, and limit sets of geometrically finite Kleinian groups," Acta Math. 153(1984), 259-277.

[33] S. Wolpert, "Thurston's Riemannian metric for Teichmüller space," J. Diff. Geom. 23(1986), $143-174$.

Boston College

UNIVERSITY OF MICHIGAN 